
stochastic neural field model

by

Matthew A. Webber

Paul C. Bressloff 



\title{
The effects of noise on binocular rivalry waves: a stochastic neural field model
}

\author{
Matthew A. Webber ${ }^{2}$ and Paul C. Bressloff ${ }^{1,2}$ \\ ${ }^{1}$ Department of Mathematics, University of Utah, 155 South 1400 East, Salt \\ Lake City UT 84112 \\ ${ }^{2}$ Mathematical Institute, University of Oxford, 24-29 St. Giles', Oxford, OX1 \\ $3 \mathrm{LB}, \mathrm{UK}$
}

\begin{abstract}
We analyse the effects of extrinsic noise on traveling waves of visual perception in a competitive neural field model of binocular rivalry. The model consists of two one-dimensional excitatory neural fields, whose activity variables represent the responses to left-eye and right-eye stimuli, respectively. The two networks mutually inhibit each other, and slow adaptation is incorporated into the model by taking the network connections to exhibit synaptic depression. We first show how, in the absence of any noise, the system supports a propagating composite wave consisting of an invading activity front in one network co-moving with a retreating front in the other network. Using a separation of time scales and perturbation methods previously developed for stochastic reaction-diffusion equations, we then show how multiplicative noise in the activity variables leads to a diffusive-like displacement (wandering) of the composite wave from its uniformly translating position at long time scales, and fluctuations in the wave profile around its instantaneous position at short time scales. The multiplicative noise also renormalizes the mean speed of the wave. We use our analysis to calculate the first passage time distribution for a stochastic rivalry wave to travel a fixed distance, which we find to be given by an inverse Gaussian. Finally, we investigate the effects of noise in the depression variables, which under an adiabatic approximation leads to quenched disorder in the neural fields during propagation of a wave.
\end{abstract}

PACS numbers: 87.19.lj

Submitted to: J. Stat. Mech.

\section{Introduction}

One of the major challenges in neurobiology is understanding the relationship between spatially structured activity states observed in both normal and pathological brain regions, and the underlying neural circuitry that supports them. This has led to considerable recent interest in studying reduced continuum neural field models in which the large-scale dynamics of spatially structured networks of neurons is described in terms of nonlinear integrodifferential equations, whose associated integral kernels represent the spatial distribution of neuronal synaptic connections [2,83,84]. Such models provide an important example of spatially extended excitable systems with nonlocal interactions. As in the case of nonlinear partial differential equation (PDE) 
models of diffusively coupled excitable systems [48,51], neural fields can exhibit a diverse range of spatiotemporal dynamics, including solitary traveling fronts and pulses, stationary pulses and spatially localized oscillations (breathers), spiral waves, and Turing-like patterns, see the reviews $[22,28,31]$. In recent years, neural fields have been used to model a wide range of neurobiological phenomena, including wave propagation in cortical slices [66,67] and in vivo [42], geometric visual hallucinations $[21,32]$, EEG rhythms $[59,64,68,76]$, orientation tuning in primary visual cortex (V1) $[6,75]$, short term working memory [25,53], control of head direction [86], and motion perception [40]. One particulary useful feature of neural fields is that analytical methods for solving these integrodifferential equations can be adapted from previous studies of nonlinear PDEs. These include regular and singular perturbation methods, weakly nonlinear analysis and pattern formation, symmetric bifurcation theory, Evans functions and wave stability, homogenization theory and averaging, and stochastic processes [22].

Recently, we have developed a continuum neural field model for binocular rivalry waves [17]. Binocular rivalry is the phenomenon whereby perception switches back and forth between different images presented to the two eyes. The resulting fluctuations in perceptual dominance and suppression provide a basis for non-invasive studies of the human visual system and the identification of possible neural mechanisms underlying conscious visual awareness $[8,9]$. Various psychophysical experiments have demonstrated that the switch between a dominant and suppressed visual percept propagates as a traveling front for each eye $[46,55,85]$. In our previous paper, we showed how such a traveling front could arise in a neural field model consisting of two mutually inhibitory, one-dimensional (1D) excitatory networks with slow adaptation [17]. The combination of cross-inhibition paired with a slow adaptive process forms the basis of most competitive network models of binocular rivalry $[47,50,53,71,72,85]$. However, these studies have neglected spatial effects or have treated them computationally. The advantage of a continuum neural field model is that it provides an analytical framework for studying perceptual wave propagation. In particular, we were able to derive an analytical expression for the speed of a binocular rivalry wave as a function of various neurophysiological parameters, and to show how properties of the wave were consistent with the wave-like propagation of perceptual dominance observed in experiments [17]. In addition to providing an analytical framework for studying binocular rivalry waves, we also showed how neural field methods provide insights into the mechanisms underlying the generation of the waves. In particular, we highlighted the important role of slow adaptation in providing a "symmetry breaking mechanism" that allows waves to propagate.

Another important feature of binocular rivalry is that it has a significant stochastic component. For example, recordings from the brain and reports by subjects during binocular rivalry tasks show dominance time statistics that may be fit to a gamma distribution [60]. In addition, statistical analysis of such data shows little

$\ddagger$ Several previous studies have modeled the spontaneous switching between rivalrous oriented stimuli in terms of a pair of ring networks (neural fields on a periodic domain) with slow adaptation and cross-inhibition, representing a pair of hypercolumns for the left and right eyes, respectively [50,54]. In these models, the rivalrous states consist of stationary activity bumps coding for either the left or right eye stimuli. (Rivalry effects in a spatially extended model have also been examined in a prior study by Loxley and Robinson [61], in which rivalrous stimuli are presented to a single onedimensional network). However, these models were not used to study binocular rivalry waves and, as formulated, were on the wrong spatial scale since they only considered short-range spatial scales comparable to a single hypercolumn (see $\S 2$ ). 
correlation between one dominance time and the next $[56,57,60]$. This suggests that the switching between one eye's dominance and the next may be largely driven by a stochastic process. Some previous models have accounted for this by presuming that the input arriving at the network encoding rivalry is stochastic, so the noise is extrinsic $[38,56]$. Recent modeling efforts have examined the effects on dominance switching of additive noise terms in either the activity or adaptation variables $[14,50,62,71]$. In this paper, we investigate the effects of noise on the propagation of binocular rivalry waves by considering a stochastic version of our neural field model. We analyze the model by extending our recent work on the theory of fluctuating fronts in neural fields with extrinsic multiplicative noise [18]. The latter, in turn, adapts methods developed previously for PDEs $[1,4,34,69]$. Such methods exploit a separation of time scales in which there is a diffusive-like displacement (wandering) of the front from its uniformly translating position at long time scales, and fluctuations in the front profile around its instantaneous position at short time scales. In the case of multiplicative noise under the Stratonovich interpretation, there is also a renormalization of the front velocity. The fact that we can carry over stochastic PDE methods to neural field equations is consistent with a number of studies that have shown how neural fields can be reduced to an equivalent PDE for particular choices of the weight kernel $[30,52,68]$.

\section{Binocular rivalry waves and the structure of $\mathrm{V} 1$}

Before introducing the basic theory of binocular rivalry waves, we first review several important properties of neurons and their functional organization within primary visual cortex (V1).

\subsection{Functional architecture of V1}

V1 is the first cortical area to receive visual information from the retina (see Fig. 1). The output from the retina is conveyed by ganglion cells whose axons form the optic nerve. The optic nerve conducts the output spike trains of the retinal ganglion cells to the lateral geniculate nucleus (LGN) of the thalamus, which acts as a relay station between retina and primary visual cortex (V1). Prior to arriving at the LGN, some ganglion cell axons cross the midline at the optic chiasm. This allows the left and right sides of the visual fields from both eyes to be represented on the right and left sides of the brain, respectively. Note that signals from the left and right eyes are segregated in the LGN and in input layers of V1. This means that the corresponding LGN and cortical neurons are monocular, in the sense that they only respond to stimuli presented to one of the eyes but not the other (ocular dominance).

One of the striking features of the visual system is that the visual world is mapped onto the cortical surface in a topographic manner. This means that neighboring points in a visual image evoke activity in neighboring regions of visual cortex. Moreover, one finds that the central region of the visual field has a larger representation in V1 than the periphery, partly due to a non-uniform distribution of retinal ganglion cells. The retinotopic map is defined as the coordinate transformation from points in the visual world to locations on the cortical surface, and can be approximated by a complex logarithm [70]. Superimposed upon the retinotopic map are additional maps reflecting the fact that neurons respond preferentially to stimuli with particular features [78]. Neurons in the retina, LGN and primary visual cortex respond to light stimuli in restricted regions of the visual field called their classical receptive fields (RFs). 
(a)

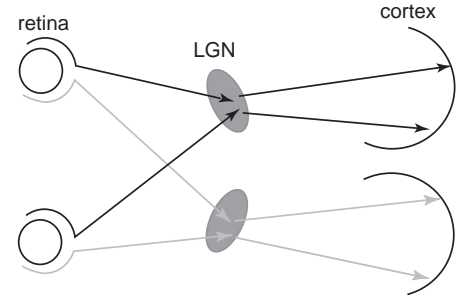

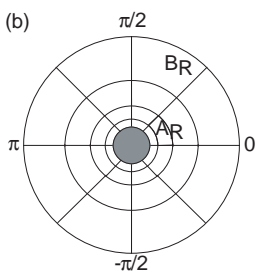

RETINA

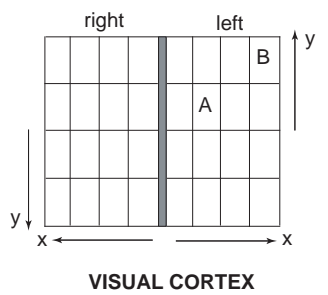

Figure 1. (a) Visual pathways from the retina through the lateral geniculate nucleus (LGN) of the thalamus to the primary visual cortex (V1). (b) Schematic illustration of the complex logarithmic mapping from retina to V1. Foveal region in retina is indicated by grey disc. Regions $A_{R}$ and $B_{R}$ in the visual field are mapped to regions $A$ and $B$ in cortex.
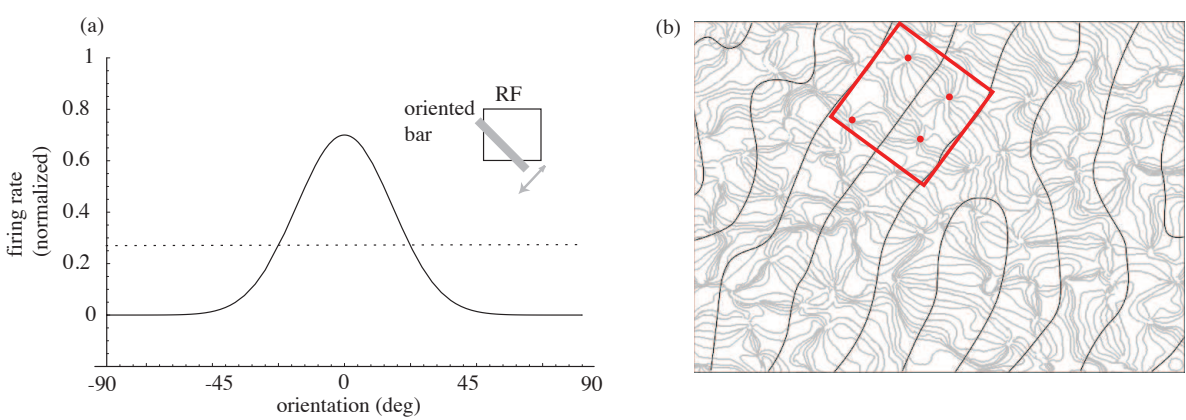

Figure 2. (a) Schematic illustration of an orientation tuning curve of a V1 neuron. Average firing rate is plotted as a function of the orientation of a bar stimulus that is moved back and forth within the receptive field (RF) of the neuron. The peak of the orientation tuning curve corresponds to the orientation preference of the cell. (b) Schematic illustration of iso-orientation (light) and ocular dominance (dark) contours in a region of primate V1. A cortical hypercolumn consistis of two orientation singularities or pinwheels per ocular dominance column.

Patterns of illumination outside the RF of a given neuron cannot generate a response directly, although they can significantly modulate responses to stimuli within the RF via long-range cortical interactions (see below). The RF is divided into distinct $\mathrm{ON}$ and $\mathrm{OFF}$ regions. In an $\mathrm{ON}$ (OFF) region illumination that is higher (lower) than the background light intensity enhances firing. The spatial arrangement of these regions determines the selectivity of the neuron to different stimuli. For example, one finds that the RFs of most V1 cells are elongated so that the cells respond preferentially to stimuli with certain preferred orientations (see Fig. 2). Similarly, the width of the ON and $\mathrm{OFF}$ regions within the RF determines the optimal spacing of alternating light and dark bars to elicit a response, that is, the cell's spatial frequency preference. In recent years much information has accumulated about the spatial distribution of orientation selective cells in V1 [41]. One finds that orientation preferences rotate smoothly over the surface of V1, so that approximately every $300 \mu \mathrm{m}$ the same preference reappears, i.e. the distribution is $\pi$-periodic in the orientation preference angle. One 
also finds that cells with similar feature preferences tend to arrange themselves in vertical columns so that to a first approximation the layered structure of cortex can be ignored. A more complete picture of the two-dimensional distribution of both orientation preference and ocular dominance in layers $2 / 3$ has been obtained using optical imaging techniques [10-12]. The basic experimental procedure involves shining light directly on to the surface of the cortex. The degree of light absorption within each patch of cortex depends on the local level of activity. Thus, when an oriented image is presented across a large part of the visual field, the regions of cortex that are particularly sensitive to that stimulus will be differentiated. The topography revealed by these methods has a number of characteristic features [65], see Fig. 2(b): (i) Orientation preference changes continuously as a function of cortical location, except at singularities or pinwheels. (ii) There exist linear zones, approximately $750 \times 750 \mu \mathrm{m}^{2}$ in area (in primates), bounded by pinwheels, within which iso-orientation regions form parallel slabs. (iii) Linear zones tend to cross the borders of ocular dominance stripes at right angles; pinwheels tend to align with the centers of ocular dominance stripes. These experimental findings suggest that there is an underlying periodicity in the microstructure of V1 with a period of approximately $1 \mathrm{~mm}$ (in cats and primates). The fundamental domain of this approximate periodic (or quasiperiodic) tiling of the cortical plane is the hypercolumn $[43,44,58]$, which contains two sets of orientation preferences $\theta \in[0, \pi)$ per eye, organized around a pair of singularities, see Fig. 2(b). Within each hypercolumn, neurons with sufficiently similar orientations tend to excite each other whereas those with sufficiently different orientations inhibit each other, and this serves to sharpen a particular neuron's orientation preference $[7,36]$. Moreover, anatomical evidence suggests that inter-hypercolumn connections link neurons with similar orientation preferences $[3,73]$.
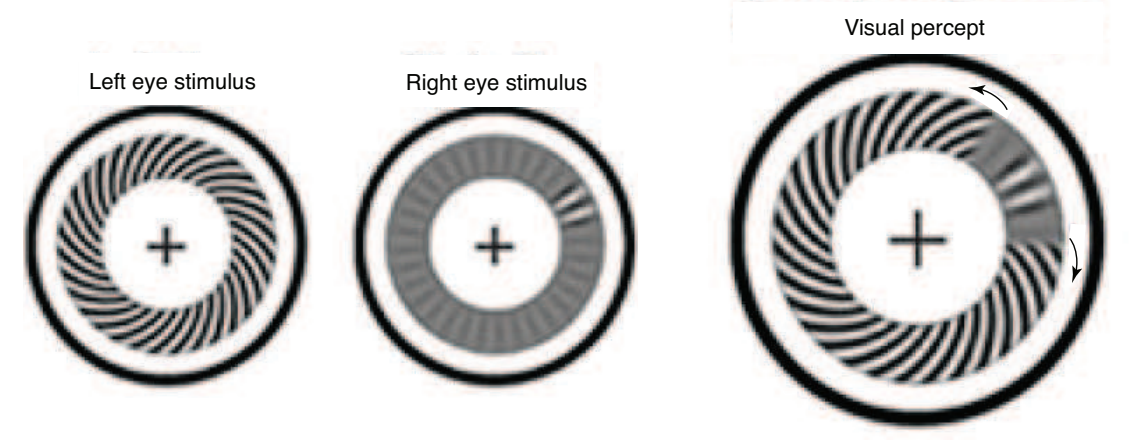

Figure 3. Illustration of a binocular wave sweeping across an annular image. To induce such a wave, a contrast increment is introduced to a specific part of the suppressed (right eye image) [85]. Figure is adapted from open-access article [26]

\subsection{Binocular rivalry waves}

One way to observe and measure the speed of perceptual waves in psychophysical experiments of binocular rivalry $[55,85]$ is to take the rival images to be a low-contrast radial grating presented to one eye and a high-contrast spiral grating presented to the other eye. Each image is restricted to an annular region of the visual field centered on the fixation point of the observer, thus effectively restricting wave propagation to 
the one dimension around the annulus. Switches in perceptual dominance can then be triggered using a brief rapid increase in stimulus contrast within a small region of the suppressed low-contrast grating. This induces a perceptual traveling wave in which the observer perceives the local dominance of the low-contrast image spreading around the annulus, see Fig. 3. The observer presses a key when the perceptual wave reaches a target area at a fixed distance from the trigger zone, and this determines the wave speed $[55,85]$. Since the rival images consist of oriented gratings, one might expect that primary visual cortex (V1) plays some role in the generation of binocular rivalry waves. Indeed, it has been shown using functional magnetic resonance imaging that there is a systematic correspondence between the spatiotemporal dynamics of activity in V1 and the time course of perceptual waves [55]. However, it has not been established whether the waves originate in V1 or are evoked by feedback from extrastriate cortex. Recently Kang et. al. [46,47] have developed a new psychophysical method for studying binocular rivalry waves that involves periodic perturbations of

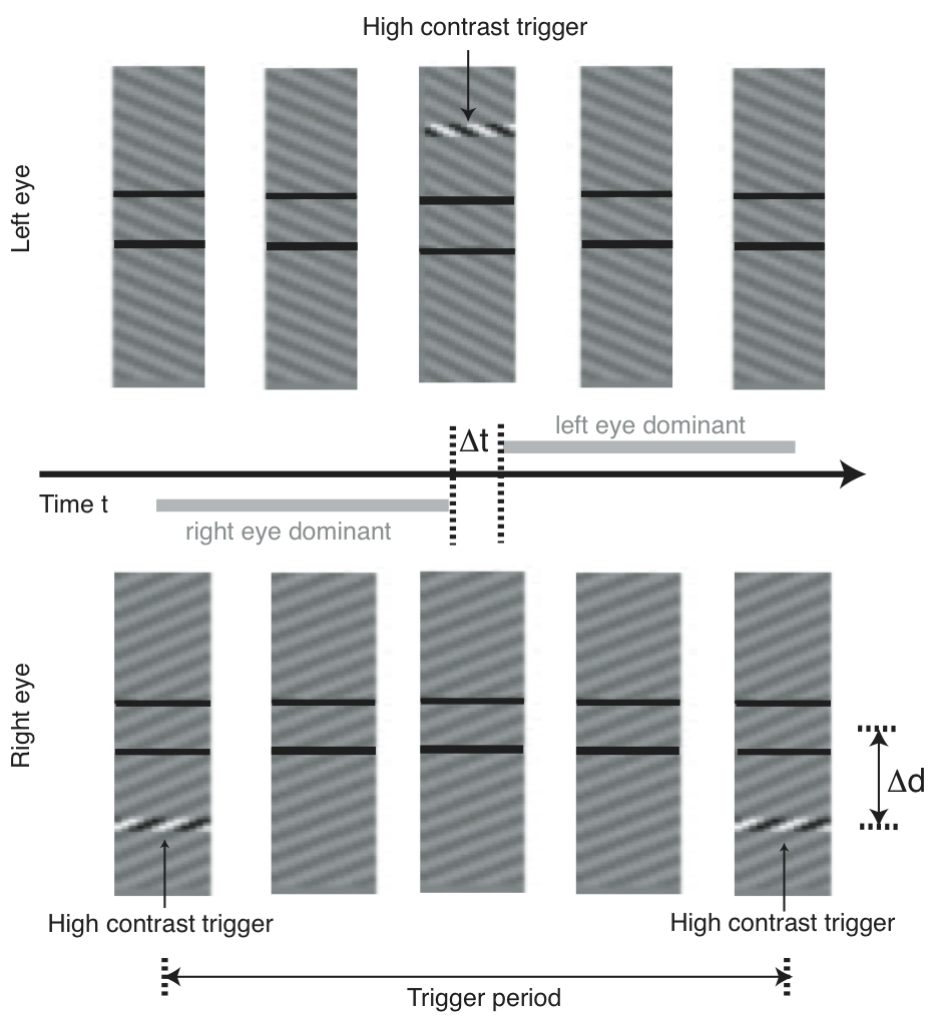

Figure 4. Schematic diagram illustrating experimental prototocol used to study binocular rivalry waves [46]. High contrast triggers are presented periodically in antiphase within the upper extended region of one grating pattern and within the lower region of the rival pattern. Subject simply reports perceptual alternations in rival dominance within the central monitoring region indicated by the horizontal black lines on each pattern. The monitoring region is a distance $\Delta d$ from the trigger region, which can be adjusted. If $\Delta t$ is the latency between the triggering event and the subsequent observation of a perceptual switch, then the speed $c$ of the wave is given by the slope of the plot $\Delta d=c \Delta t$. 
the rival images and linear gratings. An observer tracks rivalry within a small, central region of spatially extended rectangular grating patterns, while alternating contrast triggers are presented repetitively in the periphery of the rival patterns. The basic experimental set up is illustrated in Fig. 4. A number of interesting results have been obtained from these studies. First, over a range of trigger frequencies, the switching between rival percepts within the central regions is entrained to the triggering events. Moreover, the optimal triggering frequency depends on the natural frequency of spontaneous switching (in the absence of triggers). Second, the latency between triggering event and perceptual switching increases approximately linearly with the distance between the triggering site and the central region being tracked by the observer, consistent with the propagation of a traveling front. Third, the speed of the traveling wave across observers covaries with the spontaneous switching rate.

2.2.1. Network nodel of rivalry oscillations The above psychophysical experiments suggest that binocular rivalry waves consist of two basic components: the switching between rivalrous left/right eye states and the propagation of the switched state across a region of cortex. Let us first focus on the switching mechanism by neglecting spatial effects. Suppose, for the sake of illustration, that a horizontally oriented grating is presented to the left eye and a vertically oriented grating is presented to the right eye. This triggers rivalry due to the combination of orientation and eye specific crossinhibition in V1. During left eye stimulus dominance, it is assumed that a group of the left eye neurons that respond to horizontal orientations are firing persistently, while right eye neurons are suppressed by cross-inhibitory connections. Of course, there may still be some low rate firing of the right eye neurons, but it will be less than the firing rate of the left eye, horizontally tuned neurons [9]. Following this, some slow adaptive process causes a switch so that right eye, vertical orientation neurons fire persistently, suppressing the left eye neurons resulting in a repetitive cycle of perceptual dominance between the left and right eye stimuli. The competitive network architecture of reciprocal inhibition paired with slow adaptation (Fig. 5) has been used extensively to model oscillations in binocular rivalry [37, 50, 54, 71, 72, 74, 80, 85]. (In some versions of the model, recurrent excitation is ommitted). In most cases a firing

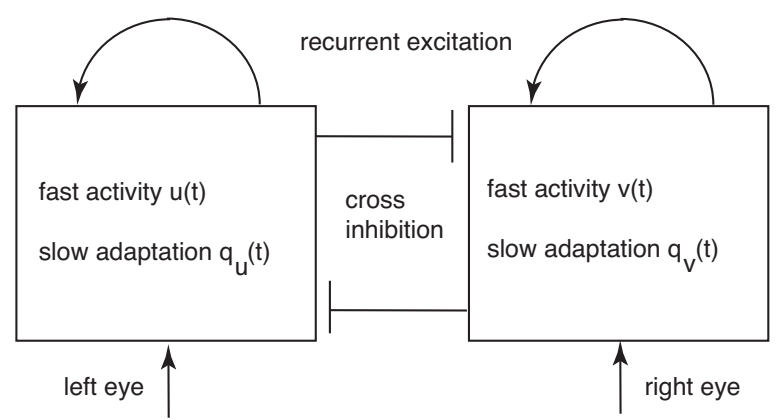

Figure 5. Schematic diagram of a competitive network architecture for rivalry oscillations $[54,71,85]$, consisting of two homogeneous populations of cells, one driven by left eye stimuli and the other by right eye stimuli. Recurrent connections within each population are assumed to be excitatory, whereas connections between the two populations are inhibitory (cross-inhibition). Each network is described by two variables, a fast activity variable and a slow adaptation variable. 
rate model appears sufficient to capture the elevation in neuronal spiking associated with the dominant stimulus.

It remains an open question as to which slow adaptive process is most responsible for the eventual switching of one stimulus dominance to the other [71]. The mechanism of spike frequency adaptation has been suggested, since it can curtail excitatory activity in a single neuron $[54,85]$. Spike frequency adaptation is the process by which a hyperpolarizing current is switched on due to a build-up of a certain ion, like calcium, within the cell due to repetitive firing [77]. The maximal firing rate of a neuron is lowered as a result. In the case of binocular rivalry, this may cause the dominant population to eventually drop its firing rate so that cross-inhibition suppressing the other population is then low enough for the suppressed populations to rekindle its firing rate into dominance. Since the recently released population is not adapted, it can then remain in dominance and suppress the other population for a period of time roughly equal to the time constant of spike frequency adaptation $[54,61,85]$. Another proposed switching mechanism is that the inhibitory synapses from one eye's neurons to the other's undergo synaptic depression§. This is the process by which synaptic resources such as neurotransmitters, vesicles, and scaffolding proteins are exhausted due to their continuous use $[27,82]$. If inhibitory synapses remain repeatedly active, due to one eye's neurons suppressing the others, eventually most of those synapses' resources will be used up, the effect of inhibition will be weakened and the suppressed population will escape $[50,54,71]$. For concreteness, we will take slow adaptation to arise from synaptic depression; however, the specific choice of adaptation does not affect the main results of our paper.

Let $u(t)$ and $v(t)$ denote the activity of the left and right eye populations at time $t$. The rate-based equations for a competitive network model with synaptic depression can be constructed as follows:

$$
\begin{aligned}
\tau \frac{d u(t)}{d t} & \left.\left.=-u(t)+I_{u}(t)+\bar{w}_{e} q_{u}(t) f(u(t))\right)-\bar{w}_{i} q_{v}(t) f(v(t))\right) \\
\tau_{s} \frac{d q_{u}(t)}{d t} & =1-q_{u}(t)-\beta q_{u}(t) f(u(t))
\end{aligned}
$$

and

$$
\begin{aligned}
\tau \frac{d v(t)}{d t} & \left.\left.=-v(t)+I_{v}(t)+\bar{w}_{e} q_{v}(t) f(v(t))\right)-\bar{w}_{i} q_{u}(t) f(u(t))\right) \\
\tau_{s} \frac{d q_{v}(t)}{d t} & =1-q_{v}(t)-\beta q_{v}(t) f(v(t)),
\end{aligned}
$$

where the positive constants $\bar{w}_{e}$ and $\bar{w}_{i}$ denote the stengths of recurrent excitatory and cross-inhibitory connections, and $I_{u}, I_{v}$ denote the input stimuli from the left and right eyes. The nonlinear function $f$ represents the mean firing rate of a local population and is usually taken to be a smooth, bounded monotonic function such as a sigmoid

$$
f(u)=\frac{1}{1+e^{-\eta(u-\kappa)}},
$$

with gain $\eta$ and threshold $\kappa$. The variables $q_{u}, q_{v}$ are taken to be synaptic depression variables representing the fraction of baseline synaptic resources that are available to the neuron for firing. These synaptic resources are depleted under continuous firing

$\S$ More precisely, synaptic depression tends to be associated only with excitatory synapses, so that in our simplified model depressing inhibitory connections would have to be mediated by excitatory connections innervating local interneurons, for example. 
at a rate proportional to $\beta q f[5,79,81]$ and recover under no firing exponentially with time constant $\tau_{s}, \tau_{s} \gg \tau$. Previously [50], we have explicitly analyzed the existence and stability of fixed point solutions of equations (2.1) and (2.4) in the high gain limit $\eta \rightarrow \infty$ of (2.5) for which $f$ becomes a Heaviside function

$$
f(u)=H(u-\kappa)= \begin{cases}0 & \text { if } u<\kappa \\ 1 & \text { if } u>\kappa .\end{cases}
$$

This choice of rate function proves particularly useful when analyzing traveling wave solutions of continuum neural fields $[2,17,29,66]$, see sections 3 and 4 . Denoting a homogeneous fixed point by $\left(U^{*}, V^{*}, Q_{u}^{*}, Q_{v}^{*}\right)$, we have for $I_{u}=I_{v}=I$ with $I$ constant,

$$
\begin{aligned}
& U^{*}=Q_{u}^{*} \bar{w}_{e} H\left(U^{*}-\kappa\right)-Q_{v}^{*} \bar{w}_{i} H\left(V^{*}-\kappa\right)+I \\
& V^{*}=Q_{v}^{*} \bar{w}_{e} H\left(V^{*}-\kappa\right)-Q_{u}^{*} \bar{w}_{i} H\left(U^{*}-\kappa\right)+I \\
& Q_{u}^{*}=\frac{1}{1+\beta H\left(U^{*}-\kappa\right)}, \quad Q_{v}^{*}=\frac{1}{1+\beta H\left(V^{*}-\kappa\right)}
\end{aligned}
$$

There are four possible homogeneous fixed points and all are stable. First, there is the off state $U^{*}=V^{*}=I$, which occurs when $I<\kappa$, that is, the input is not strong enough to activate either population. Second there is the on-state or fusion state, where both populations are simultaneously active:

$$
\begin{aligned}
& \left(U^{*}, V^{*}\right)=\left(\frac{\bar{w}_{e}-\bar{w}_{i}}{1+\beta}+I, \frac{\bar{w}_{e}-\bar{w}_{i}}{1+\beta}+I\right), \\
& \left(Q_{u}^{*}, Q_{v}^{*}\right)=\left(\frac{1}{1+\beta}, \frac{1}{1+\beta}\right),
\end{aligned}
$$

and occurs when $I>\kappa-\left(\bar{w}_{e}-\bar{w}_{i}\right) /(1+\beta)$. This case is more likely for very strong depression ( $\beta$ large), since cross inhibition will be weak, or when the local connections are strong and excitation-dominated. Finally there are two winner-take-all (WTA) states in which one population dominates the other: the left eye dominant state

$$
\begin{aligned}
& \left(U^{*}, V^{*}\right)=\left(\frac{\bar{w}_{e}}{1+\beta}+I, I-\frac{\bar{w}_{i}}{1+\beta}\right), \\
& \left(Q_{u}^{*}, Q_{v}^{*}\right)=\left(\frac{1}{1+\beta}, 1\right)
\end{aligned}
$$

and the right eye dominant state

$$
\begin{aligned}
& \left(U^{*}, V^{*}\right)=\left(I-\frac{\bar{w}_{i}}{1+\beta}, \frac{\bar{w}_{e}}{1+\beta}+I\right), \\
& \left(Q_{u}^{*}, Q_{v}^{*}\right)=\left(1, \frac{1}{1+\beta}\right)
\end{aligned}
$$

The WTA states exist provided that

$$
I>\kappa-\frac{\bar{w}_{e}}{1+\beta}, \quad I<\kappa+\frac{\bar{w}_{i}}{1+\beta}
$$

This will occur in the presence of weak depression ( $\beta$ small $)$ and strong cross-inhibition such that depression cannot exhaust the dominant hold one population has on the other. It can also be shown that equations (2.1)-(2.4) also support homogeneous limit cycle oscillations in which there is periodic switching between left and right eye dominance consistent with binocular rivalry [50]. Since all the fixed points are stable, it follows that such oscillations cannot arise via a standard Hopf bifurcation. Indeed, we find bistable regimes where a rivalry state coexists with a fusion state as illustrated in Fig. 6. (Such behavior persists in the case of smooth sigmoid firing rate functions, at least for sufficiently high gain [50]). 

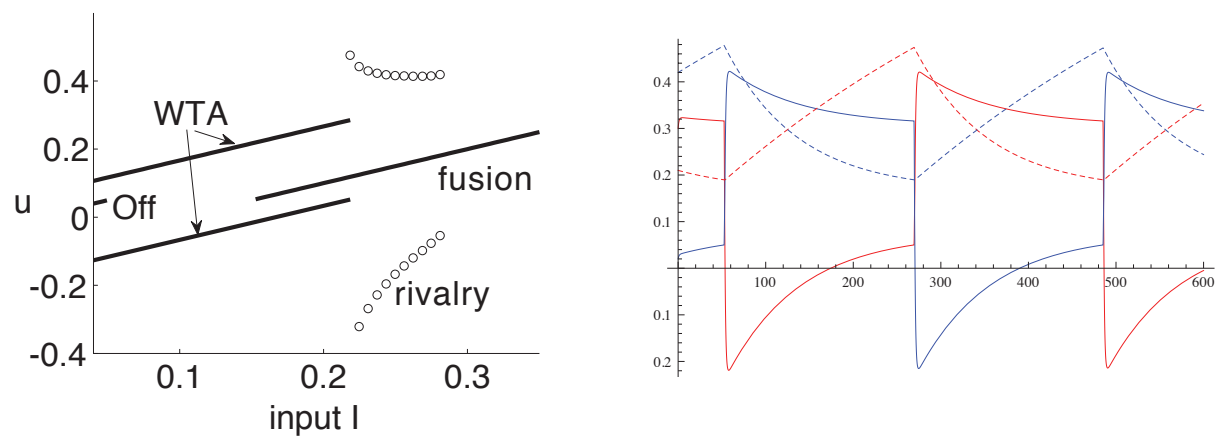

Figure 6. (a) Bifurcation diagram showing homogeneous solutions for the left population activity $u$ as a function of the input amplitude $I$. Solid lines represent stable states, whereas circles represent the maximum and minimum of rivalry oscillations. It can be seen that there are regions of off/WTA bistability, WTA/fusion bistability, and fusion/rivalry bistability. Parameters are $\tau_{s}=500$, $\kappa=0.05, \beta=5, \bar{w}_{e}=0.4$ and $\bar{w}_{i}=1$. (b) Homogeneous oscillatory solution in which there is spontaneous periodic switching between left and right eye dominance. Plot against time of the left eye neural activity $u$ (solid red), the right eye neural activity $v$ (solid blue) together with the corresponding depression variables $q_{u}$ (dashed red) and $q_{v}$ (dashed blue).

2.2.2. Network model of rivalry waves In order to take into account the propagation of activity seen in binocular rivalry waves, it is necessary to introduce a spatially extended network model. Therefore, suppose that the neuronal populations responding to stimuli from the left-eye, say, are distributed on a one-dimensional (1D) lattice and are labeled according to the integer $n$; a second 1D network responds to stimuli from the right eye, see Fig. 7. In terms of the functional architecture of $\mathrm{V} 1$, one can interpret the $n$th left/right eye populations as consisting of neurons in a given hypercolumn that respond maximally to the distinct stimuli presented to the two eyes at a particular coarse-grained location in space. In the case of the orientated grating stimuli used by Kang et. al. [46, 47], see Fig. 4, this would mean neurons whose orientation preference coincides with the stimulus orientation presented to a given eye. Since the orientation does not change along the length of the grating, all neurons receive the same external drive. Letting $u_{n}, v_{n}, q_{u, n}, q_{v, n}$ denote the activity and depression variables of the $n$th left and right eye networks, we have

$\left.\left.\left.\tau \frac{d u_{m}}{d t}=-u_{m}+I_{u}+\sum_{n}\left[w_{e}\right]_{m n} q_{u, n} f\left(u_{n}\right)\right)-\left[w_{i}\right]_{m n} q_{v, n} f\left(v_{n}\right)\right)\right]$

$\tau_{s} \frac{d q_{u, m}}{d t}=1-q_{u, m}-\beta q_{u, m} f\left(u_{m}\right)$

and

$\tau \frac{d v_{m}}{d t}=-v_{m}+I_{v}+\sum_{n}\left(\left[w_{e}\right]_{m n} q_{v, n} f\left(v_{n}\right)-\left[w_{i}\right]_{m n} q_{u, n} f\left(u_{n}\right)\right)$

$\tau_{s} \frac{d q_{v, m}}{d t}=1-q_{v, m}-\beta q_{v, m} f\left(v_{m}\right)$.

Here $\left[w_{e}\right]_{m n}$ is the strength of excitation from the $n$th to the $m$ th population with the same eye preference, and $\left[w_{i}\right]_{m n}$ is the strength of cross-inhibition between populations 
with opposite eye preferences. The weights are typically assumed to decrease with distance of separation $|m-n|$ according to an exponential or Gaussian distribution. Slow adaptation is incorporated into the model by taking the network connections to exhibit synaptic depression along the lines of Kilpatrick and Bressloff [50]. Note that a similar network architecture was previously considered in a computational model of binocular rivalry waves $[47,85]$, in which cross inhibition was mediated explicitly by interneurons and, rather than including depressing synapses, the excitatory neurons were taken to exhibit spike frequency adaptation. Numerical simulations of the model showed that the network supported traveling waves consistent with those observed numerically under physiologically reasonable parameter regimes.

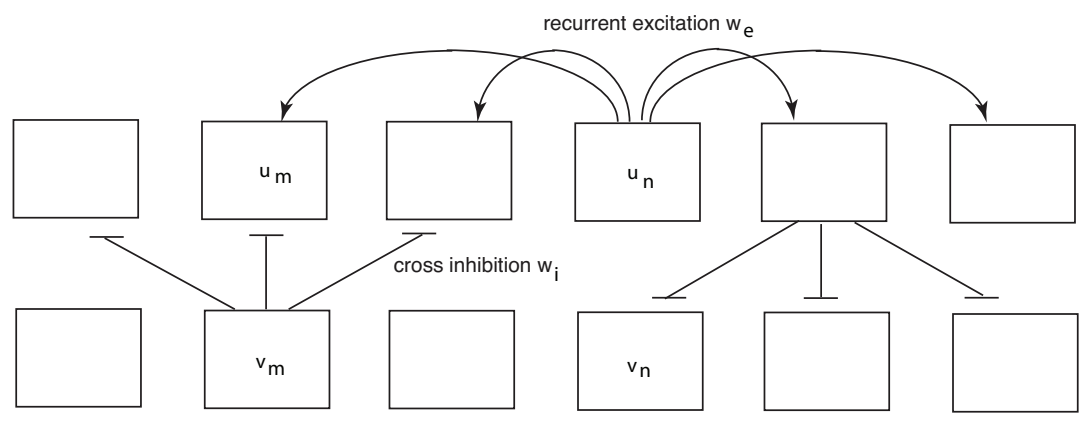

Figure 7. Schematic diagram of a competitive neural network consisting of two sets of populations distributed along a 1D lattice Recurrent connections within each 1D network are assumed to be excitatory, whereas connections between the two networks are inhibitory (cross-inhibition). Slow adaptation is incorporated into the model by taking the network connections to exhibit synaptic depression.

\section{Stochastic neural field model of binocular rivalry waves}

Although the discrete lattice model presented in section 2.2 .2 is directly amenable to numerical simulations, it is difficult to derive any analytical results for the model that generate, for example, explicit expressions for how the wavespeed depends on network parameters. Therefore, in this section we turn to a neural field model that can be constructed by taking an appropriate continuum limit of equations (2.7) and (2.10). Previously, we analyzed the existence and stability of traveling front solutions (rivalry waves) in such a model [22]. Here we show how a theory of fluctuating fronts in stochastic neural fields, see [18], can be used to analyze the effects of noise on binocular rivalry waves.

\subsection{Rivalry waves in a deterministic neural field model}

We begin by considering the determinsitic neural field model analyzed in [22]. This can be interpreted as a continuum version of the discrete network model of Fig. 7 . Introduce a lattice spacing $a$, and write $\left[w_{e}\right]_{n m}=a w_{e}(n a, m a), u_{n}(t)=u(n a, t)$ etc. Substituting into equations (2.7)-(2.10) and taking the continuum limit $a \rightarrow 0$ such that $n a \rightarrow x$ and $m a \rightarrow y$, the discrete sums can be replaced by integrals to give the neural field model

$$
\left.\tau \frac{d u(x, t)}{d t}=-u(x, t)+I_{u}+\int_{-\infty}^{\infty} w_{e}\left(x-x^{\prime}\right) q_{u}\left(x^{\prime}, t\right) f\left(u\left(x^{\prime}, t\right)\right)\right) d x^{\prime}
$$


Stochastic neural field model of binocular rivalry waves

$$
\begin{aligned}
& \left.-\int_{-\infty}^{\infty} w_{i}\left(x-x^{\prime}\right) q_{v}\left(x^{\prime}, t\right) f\left(v\left(x^{\prime}, t\right)\right)\right) d x^{\prime} \\
\tau_{s} \frac{d q_{u}(x, t)}{d t}= & 1-q_{u}(x, t)-\beta q_{u}(x, t) f(u(x, t))
\end{aligned}
$$

and

$$
\begin{aligned}
\tau \frac{d v(x, t)}{d t}= & \left.-v(x, t)+I_{v}+\int_{-\infty}^{\infty} w_{e}\left(x-x^{\prime}\right) q_{v}\left(x^{\prime}, t\right) f\left(v\left(x^{\prime}, t\right)\right)\right) d x^{\prime} \\
& \left.-\int_{-\infty}^{\infty} w_{i}\left(x-x^{\prime}\right) q_{u}\left(x^{\prime}, t\right) f\left(u\left(x^{\prime}, t\right)\right)\right) d x^{\prime} \\
\tau_{s} \frac{d q_{v}(x, t)}{d t}= & 1-q_{v}(x, t)-\beta q_{v}(x, t) f(v(x, t))
\end{aligned}
$$

assuming that the weights only depend on the distance between interacting populations. For concreteness, the distributions $w_{e}$ and $w_{i}$ are both taken to be Gaussians:

$$
w_{e}(r)=\frac{\bar{w}_{e}}{\sqrt{2 \pi \sigma_{e}^{2}}} e^{-\frac{r^{2}}{2 \sigma_{e}^{2}}}, \quad w_{i}(r)=\frac{\bar{w}_{i}}{\sqrt{2 \pi \sigma_{i}^{2}}} e^{-\frac{r^{2}}{2 \sigma_{i}^{2}}} .
$$

Following [22], we assume that $\sigma_{e}>\sigma_{i}$ (longer range excitation) and fix the length scale by setting $\sigma_{e}=2, \sigma_{i}=1$. Assuming that excitation spans a single hypercolumn, $\sigma_{e}$ should be the same approximate size as a hypercolumn, that is, of the order $200 \mu \mathrm{m}$. We also take $\tau=1$ in units of the membrane time constantt, which is typically of the order $10 \mathrm{msec}$.

The next step is to interpret the binocular rivalry wave seen in the experiments of Kang et. al. $[46,47]$ as a traveling wave front solution of the neural field equations (3.1)-(3.4), in which a high activity state invades the suppressed left eye network, say, whilst retreating from the dominant right eye network, see Fig. 8. Such a wave is defined as

$$
u(x, t)=U(x-c t), \quad v(x, t)=V(x-c t)
$$

where $c$ is the wave speed and $\xi=x-c t$ is a traveling wave coordinate together with the asymptotic conditions

$$
\begin{aligned}
& (U(\xi), V(\xi)) \rightarrow \mathbf{X}_{L} \text { as } \xi \rightarrow-\infty, \\
& (U(\xi), V(\xi)) \rightarrow \mathbf{X}_{R} \text { as } \xi \rightarrow \infty
\end{aligned}
$$

with $U(\xi)$ a monotonically decreasing function of $\xi$ and $V(\xi)$ a montonically increasing function of $\xi$. Here $\mathbf{X}_{L}\left(\mathbf{X}_{R}\right)$ represents a homogeneous left-eye (right-eye) dominant state. Given the asymptotic behavior of the solution and the requirements of monotonicity, we see that $U(\xi)$ and $V(\xi)$ each cross threshold at a single location, which may be different for the two eyes. Exploiting translation invariance we take $U(0)=\kappa$ and $V(X)=\kappa$.

As we showed in our previous paper [22], it is possible to construct an exact traveling wave solution of equations $(3.1)-(3.4)$ by taking $f$ to be the Heaviside function (2.6) and making the adiabatic approximation that $q_{u}(x, t)=Q_{u}, q_{v}(x, t)=$ $Q_{v}$ with $Q_{u}, Q_{v}$ constants. The latter is based on the assumption that adaptation is sufficiently slow so that the wave traverses cortex in a time $T_{l} \ll \tau_{s}$. Substituting the traveling wave solution (3.6) into equations (3.1) and (3.3) with fixed $Q_{u}, Q_{v}$ and 


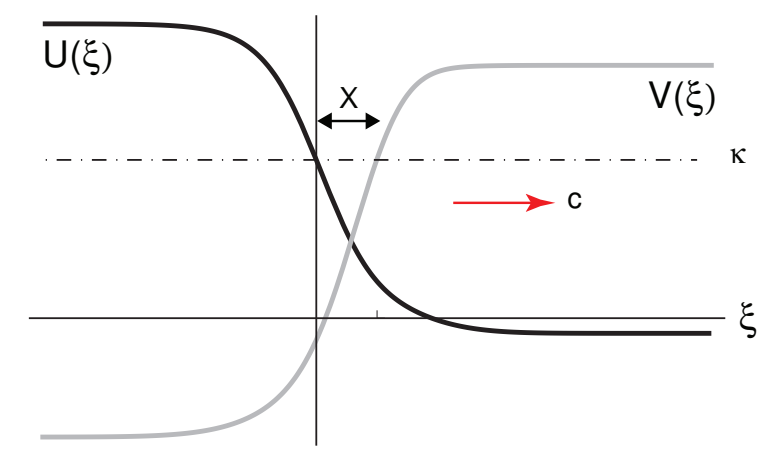

Figure 8. Sketch of a right-moving traveling wave solution in which a high activity state invades the suppressed left eye network whilst retreating from the dominant right eye network. Wave is shown in a moving frame $\xi=x-c t$.

$f(u)=H(u-\kappa)$ leads to the equations

$$
\begin{aligned}
& -c \frac{d U}{d \xi}+U=Q_{u} \int_{-\infty}^{0} w_{e}\left(\xi-\xi^{\prime}\right) d \xi^{\prime}-Q_{v} \int_{X}^{\infty} w_{i}\left(\xi-\xi^{\prime}\right) d \xi^{\prime}+I \\
& -c \frac{d V}{d \xi}+V=Q_{v} \int_{X}^{\infty} w_{e}\left(\xi-\xi^{\prime}\right) d \xi^{\prime}-Q_{u} \int_{-\infty}^{0} w_{i}\left(\xi-\xi^{\prime}\right) d \xi^{\prime}+I .
\end{aligned}
$$

where, for simplicity, we have set $I_{u}=I_{v}=I$. Multiplying both sides of equations (3.8) and (3.9) by $\mathrm{e}^{-\xi / c}$, integrating with respect to $\xi$ and imposing the threshold conditions gives

$$
U(\xi)=\mathrm{e}^{\xi / c}\left[\kappa-\frac{1}{c} \int_{0}^{\xi} \mathrm{e}^{-z / c} \Psi_{X}(z) d z-I\left(1-\mathrm{e}^{-\xi / c}\right)\right], \xi>0
$$

and

$V(\xi)=\mathrm{e}^{(\xi-X) / c}\left[\kappa-\frac{1}{c} \int_{0}^{\xi-X} \mathrm{e}^{-z / c} \Phi_{X}(-z) d z-I\left(1-\mathrm{e}^{-(\xi-X) / c}\right)\right], \xi>X$

with $\Psi$ and $\Phi$ defined by

$$
\begin{aligned}
& \Psi_{X}(z)=Q_{u} \int_{z}^{\infty} w_{e}(y) d y-Q_{v} \int_{-\infty}^{z-X} w_{i}(y) d y \\
& \Phi_{X}(z)=Q_{v} \int_{z}^{\infty} w_{e}(y) d y-Q_{u} \int_{-\infty}^{z-X} w_{i}(y) d y
\end{aligned}
$$

Finally, requiring that the wave solution remain bounded as $\xi \rightarrow \infty$ (assuming $c>0$ ), yields the pair of threshold conditions

$$
\kappa=\int_{0}^{\infty} \mathrm{e}^{-s} \Psi_{X}(c s) d s+I, \quad \kappa=\int_{0}^{\infty} \mathrm{e}^{-s} \Phi_{X}(-c s) d s+I .
$$

In the particular case $Q_{u}=Q_{v}=1$ (no synaptic depression), it can be shown that the above equations have no solution for $c \neq 0$, that is, there does not exists a traveling wave solution [17]. On the other hand, slow synaptic depression with $Q_{u} \neq Q_{v}$ breaks the symmetry of the threshold crossing conditions, leading to a unique solution for $c, X$ as a function of the network parameters. Note that one can also establish that 
the traveling front is linearly stable [22]. The threshold conditions can also be used to simlify the expressions for the wave profile, namely,

$$
U(\xi)=\frac{1}{c} \int_{0}^{\infty} \mathrm{e}^{-z / c} \Psi_{X}(z+\xi) d z+I
$$

and

$$
V(\xi)=\frac{1}{c} \int_{0}^{\infty} \mathrm{e}^{-z / c} \Phi_{X}(-z-\xi+X) d z+I .
$$

Example plots of the wavespeed are shown in Fig. 9. Baseline parameter values are chosen so that spontaneous oscillations and traveling fronts co-exist as found experimentally $[46,47]$. The model wave speed is of the order $c=1$ in dimensionless units, that is, $c=\sigma_{e} / 2 \tau$ where $\sigma_{e}$ is the range of excitation and $\tau$ is the membrane time constant. Taking $\sigma_{e}$ to be of the order $200 \mu \mathrm{m}$ and $\tau$ to be of the order 10 msec yields a wavespeed of around $10 \mathrm{~mm} / \mathrm{sec}$, which is consistent with the speeds observed experimentally. (In the psychophysical experiments of Kang et. al., binocular rivalry waves took approximately 0.8 seconds to traverse 2 degrees of the visual field. The magnification factor in humans throughout the foveal region is approximately $0.4 \mathrm{~cm} / \mathrm{deg}$, which corresponds to $0.8 \mathrm{~cm}$ of cortex). Fig. 9 shows that the speed of the wave is a decreasing function of the threshold $\kappa$ and an increasing function of the input amplitude $I$; the latter is consistent with what is found experimentally when the stimulus contrast is increased $[46,47]$. Yet another experimental result that emerges from the model is that the wavespeed covaries with the frequency of spontaneous rivalry oscillations [22].
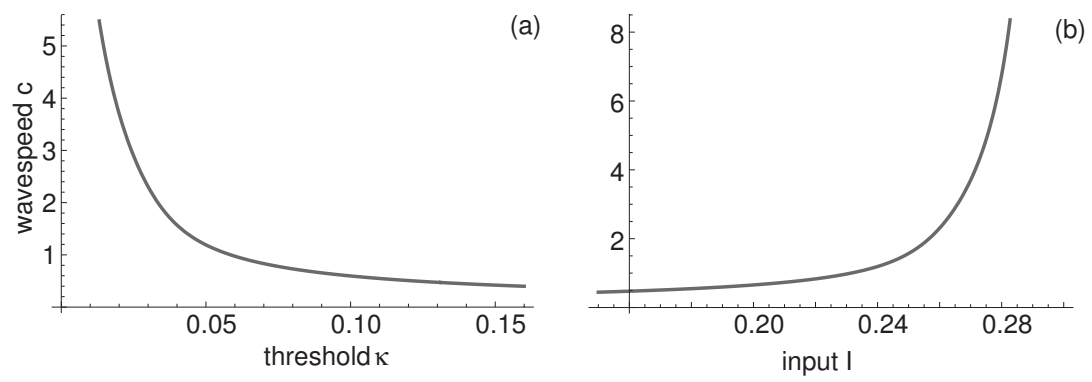

Figure 9. Plot of wave speed $c$ in units of $\sigma_{2} / 2 \tau$ ) as a function of (a) the threshold $\kappa$ and (b) the external input strength $I$. The default parameters are taken to be $\bar{w}_{i}=1, \bar{w}_{e}=0.4, \sigma_{i}=1, \sigma_{e}=2, \beta=5, \kappa=0.05, I=0.24, Q_{u}=0.42, Q_{v}=0.25$ and the corresponding wave speed is $c=1.2$. For this set of parameters, the network operates in a regime that supports both traveling waves and homogeneous oscillatory solutions, that is, spontaneous switching occurs in the absence of traveling waves.

\subsection{Effects of multiplicative noise in the fast activity variables}

Several recent studies have considered stochastic versions of neural field equations that are based on a corresponding Langevin equation formulation [13, 18, 35, 45]. Motivated by these examples and the adiabatic approximation used in the analysis of deterministic waves, we consider the following Langevin equation (or stochastic PDE) 
for the stochastic activity variables $U(x, t)$ and $V(x, t)$ :

$$
\begin{aligned}
d U= & {\left[-U+Q_{u} \int w_{e}(x-y) f \circ U(y, t) d y-Q_{v} \int w_{i}(x-y) f \circ V(y, t) d y+I_{u}\right] d t } \\
& +\epsilon^{\frac{1}{2}} g(U) \circ d W_{u} \\
d V= & {\left[-V+q_{v} \int w_{e}(x-y) f \circ V(y, t) d y-Q_{u} \int w_{i}(x-y) f \circ U(y, t) d y+I_{v}\right] d t } \\
& +\epsilon^{\frac{1}{2}} g(V) \circ d W_{v} .
\end{aligned}
$$

with $Q_{u}, Q_{v}$ fixed. We assume that $W_{u}, W_{v}$ represent independent Wiener processes such that

$$
\left\langle d W_{\{u, v\}}(x, t)\right\rangle=0, \quad\left\langle d W_{i}(x, t) d W_{j}\left(x^{\prime}, t^{\prime}\right)\right\rangle=2 \delta_{i, j} C\left(\left[x-x^{\prime}\right] / \lambda\right) \delta\left(t-t^{\prime}\right) d t d t^{\prime},
$$

where $i, j=u, v$ and $\langle\cdot\rangle$ denotes averaging with respect to the Wiener processes. Here $\lambda$ is the spatial correlation length of the noise such that $C(x / \lambda) \rightarrow \delta(x)$ in the limit $\lambda \rightarrow 0$, and $\epsilon$ determines the strength of the noise, which is assumed to be weak. Following standard formulations of Langevin equations [39], the multiplicative noise term is taken to be of Stratonovich form in the case of extrinsic noise. Note, however, that an alternative formulation of stochastic neural field theory has been developed in terms of a neural master equation $[15,16,23,24]$, in which the underlying deterministic equations are recovered in the thermodynamic limit $N \rightarrow \infty$, where $N$ is a measure of the system size of each local population. In the case of large but finite $N$, a KramersMoyal expansion of the master equation yields a Langevin neural field equation with multiplicative noise of the Ito form $[15,16]$.

The effects of multiplicative noise on binocular rivalry waves can be analyzed using methods previously developed for reaction-diffusion equations $[1,4,34,69]$, which we recently extended to scalar neural field equations [18]. The starting point of such methods is the observation that multiplicative noise in the Stratonovich sense leads to a systematic shift in the speed of the front (assuming a front of speed $c$ exists when $\epsilon=0)$. This is a consequence of the fact that $\left\langle g(U) d W_{u}\right\rangle \neq 0$ and $\left\langle g(V) d W_{v}\right\rangle \neq 0$ even though $\left\langle d W_{\{u, v\}}\right\rangle=0$. These averages can be calculated using Novikov's theorem [63]:

$$
\left\langle g(U) d W_{u}\right\rangle=\epsilon^{1 / 2} C(0)\left\langle g^{\prime}(U) g(U)\right\rangle d t, \quad\left\langle g(V) d W_{v}\right\rangle=\epsilon^{1 / 2} C(0)\left\langle g^{\prime}(V) g(V)\right\rangle d t
$$

An alternative way to derive the above result is to Fourier transform equation (3.17) and evaluate averages using the corresponding Fokker-Planck equation in Fourier space (see [69] and appendix of [18]). Note that in the limit $\lambda \rightarrow 0, C(0) \rightarrow 1 / \Delta x$ where $\Delta x$ is a lattice cut-off, which can be identified with the step size of the spatial discretization scheme used in numerical simulations. Following $[4,18]$, it is convenient to rewrite equations (3.17) and (3.18) so that the fluctuating term has zero mean:

$$
\begin{aligned}
d U= & {\left[h(U)+Q_{u} \int w_{e}(x-y) f \circ U(y, t) d y-Q_{v} \int w_{i}(x-y) f \circ V(y, t) d y+I_{u}\right] d t } \\
& +\epsilon^{\frac{1}{2}} d R_{u}(U, x, t) \\
d V= & {\left[h(V)+Q_{v} \int w_{e}(x-y) f \circ V(y, t) d y-Q_{u} \int w_{i}(x-y) f \circ U(y, t) d y+I_{v}\right] d t } \\
& +\epsilon^{\frac{1}{2}} d R_{v}(V, x, t),
\end{aligned}
$$

where

$$
h(U)=-U+\epsilon C(0) g^{\prime}(U) g(U),
$$


and

$$
\begin{aligned}
& d R_{u}(U, x, t)=g(U) \circ d W_{u}-\epsilon^{\frac{1}{2}} C(0) g(U) g^{\prime}(U) d t \\
& d R_{v}(V, x, t)=g(V) \circ d W_{v}-\epsilon^{\frac{1}{2}} C(0) g(V) g^{\prime}(V) d t
\end{aligned}
$$

The stochastic processes $R_{u}, R_{v}$ have zero mean (so do not contribute to the effective drift, that is, the average wave speed) and covariance

$$
\begin{aligned}
& \left\langle d R_{u}(U, x, t) d R_{u}\left(U, x^{\prime}, t\right)\right\rangle=\left\langle g(U(x, t)) d W_{u}(x, t) g\left(U\left(x^{\prime}, t\right) d W_{u}\left(x^{\prime}, t\right)\right\rangle+\mathcal{O}\left(\epsilon^{1 / 2}\right),\right. \\
& \left\langle d R_{v}(V, x, t) d R_{v}\left(V, x^{\prime}, t\right)\right\rangle=\left\langle g(V(x, t)) d W_{v}(x, t) g\left(V\left(x^{\prime}, t\right) d W_{v}\left(x^{\prime}, t\right)\right\rangle+\mathcal{O}\left(\epsilon^{1 / 2}\right)\right. \\
& \left\langle d R_{u}(U, x, t) d R_{v}\left(V, x^{\prime}, t\right)\right\rangle=\left\langle g(U(x, t)) d W_{u}(x, t) g\left(V\left(x^{\prime}, t\right) d W_{v}\left(x^{\prime}, t\right)\right\rangle+\mathcal{O}\left(\epsilon^{1 / 2}\right) .\right.
\end{aligned}
$$

The next step in the analysis is to assume that the fluctuating terms in equations (3.21) and (3.22) generate two distinct phenomena that occur on different timescales: a diffusive-like displacement of the binocular rivalry wave from its uniformly translating position at long time scales, and fluctuations in the wave profile around its instantaneous position at short time scales $[1,4,18,34,69]$. It is important to point out that, in contrast to traveling front solutions of scalar neural field equations [18], we are now considering composite wave solutions consisting of an invading front in the left eye network, say, comoving with a retreating front in the right eye network. Thus in addition to the center-of-mass of the composite wave, which moves with speed $c$ in the absence of noise, there is an additional degree of freedom corresponding to the "width" of the composite wave. (In the case of a Heaviside rate function, the width is determined by the threshold crossing point $X$, see equation (3.14). For simplicitly, we assume that the width of the composite wave is only weakly affected by the noise; this is consistent with what is found numerically. We now express the solution $(U, V)$ of equations (3.21) and (3.22) as a combination of a fixed wave profile $\left(U_{0}, V_{0}\right)$ that is displaced by an amount $\Delta(t)$ from its uniformly translating position $\xi=x-c_{\epsilon} t$ and a time-dependent fluctuation $\left(U_{1}, V_{1}\right)$ in the wave shape about its instantaneous position:

$$
\begin{aligned}
& U(x, t)=U_{0}(\xi-\Delta(t))+\epsilon^{1 / 2} U_{1}(\xi-\Delta(t), t), \\
& V(x, t)=V_{0}(\xi-\Delta(t))+\epsilon^{1 / 2} V_{1}(\xi-\Delta(t), t) .
\end{aligned}
$$

The wave profile $\left(U_{0}, V_{0}\right)$ and associated wave speed $c_{\epsilon}$ are obtained by solving the modified deterministic equation

$$
\begin{gathered}
-c \frac{d U_{0}}{d \epsilon}-h\left(U_{0}\right)=Q_{u} \int_{-\infty}^{\infty} w_{e}\left(\xi-\xi^{\prime}\right) f \circ U_{0}\left(\xi^{\prime}\right) d \xi^{\prime}-Q_{v} \int_{-\infty}^{\infty} w_{i}\left(\xi-\xi^{\prime}\right) f \circ V_{0}\left(\xi^{\prime}\right) d \xi^{\prime} \\
+I_{u} \\
-c \frac{d V_{0}}{d \epsilon}-h\left(V_{0}\right)=Q_{v} \int_{-\infty}^{\infty} w_{e}\left(\xi-\xi^{\prime}\right) f \circ V_{0}\left(\xi^{\prime}\right) d \xi^{\prime}-Q_{u} \int_{-\infty}^{\infty} w_{i}\left(\xi-\xi^{\prime}\right) f \circ U_{0}\left(\xi^{\prime}\right) d \xi^{\prime} \\
+I_{v}
\end{gathered}
$$

Both $c_{\epsilon}$ and $U_{0}$ depend non-trivially on the noise strength $\epsilon$ due to the $\epsilon$-dependence of the function $h$, see equation (3.23). Thus, $c_{\epsilon} \neq c$ for $\epsilon>0$ and $c_{0}=c$, where $c$ is the speed of the front in the absence of multiplicative noise. It also follows that the expansions (3.26) and (3.27) are not equivalent to a standard small-noise expansion in $\epsilon$. Equations (3.28) and (3.29) are chosen so that that to leading order, the stochastic variable $\Delta(t)$ undergoes unbiased Brownian motion with a diffusion coefficient $D(\epsilon)=\mathcal{O}(\epsilon)$ (see below). Thus $\Delta(t)$ represents the effects of slow fluctuations, whereas $\left(U_{1}, V_{1}\right)$ represents the effects of fast fluctuations. 
The next step is to substitute the decompositions (3.26) and (3.27) into equations (3.21) and (3.22) and expand to first order in $\mathcal{O}\left(\varepsilon^{1 / 2}\right)$ :

$$
\begin{aligned}
-\left[c_{\varepsilon}+\right. & \dot{\Delta}] U_{0}^{\prime}\left(\xi_{\Delta}\right) d t+\epsilon^{1 / 2}\left[d U_{1}\left(\xi_{\Delta}, t\right)-\left[c_{\epsilon}+\dot{\Delta}\right] U_{1}^{\prime}\left(\xi_{\Delta}, t\right) d t\right] \\
= & h\left(U_{0}\left(\xi_{\Delta}\right)\right) d t+\epsilon^{1 / 2} h^{\prime}\left(U_{0}\left(\xi_{\Delta}\right)\right) U_{1}\left(\xi_{\Delta}, t\right) d t \\
& +Q_{u} \int_{-\infty}^{\infty} w_{e}\left(\xi-\xi^{\prime}\right)\left(f\left(U_{0}\left(\xi_{\Delta}^{\prime}\right)\right)+\epsilon^{1 / 2} f^{\prime}\left(U_{0}\left(\xi_{\Delta}^{\prime}\right)\right) U_{1}\left(\xi_{\Delta}^{\prime}, t\right)\right) d \xi^{\prime} d t \\
& -Q_{v} \int_{-\infty}^{\infty} w_{i}\left(\xi-\xi^{\prime}\right)\left(f\left(V_{0}\left(\xi_{\Delta}^{\prime}\right)\right)+\epsilon^{1 / 2} f^{\prime}\left(V_{0}\left(\xi_{\Delta}^{\prime}\right)\right) V_{1}\left(\xi_{\Delta}^{\prime}, t\right)\right) d \xi^{\prime} d t \\
& +\epsilon^{1 / 2} d R\left(U_{0}\left(\xi_{\Delta}\right), \xi, t\right)+\mathcal{O}(\epsilon) .
\end{aligned}
$$

where we have set $\xi_{\Delta}=\xi-\Delta(t)$ and $\xi_{\Delta}^{\prime}=\xi^{\prime}-\Delta(t)$. A similar equation holds for $d V$. We now use the steady state equations (3.28) and (3.29) for $U_{0}, V_{0}$, after shifting $\xi \rightarrow \xi-\Delta(t)$, to eliminate terms and then divide through by $\sqrt{\epsilon}$. This gives the inhomogeneous equations to $\mathcal{O}\left(\epsilon^{1 / 2}\right)$

$$
\begin{aligned}
& d U_{1}\left(\xi_{\Delta}, t\right)-L_{u}\left(U_{1}\left(\xi_{\Delta}, t\right), V_{1}\left(\xi_{\Delta}, t\right)\right)=\epsilon^{-\frac{1}{2}} U_{0}^{\prime}\left(\xi_{\Delta}\right) d \Delta(t)+d R_{u}\left(U_{0}\left(\xi_{\Delta}\right), \xi, t\right) \\
& d V_{1}\left(\xi_{\Delta}, t\right)-L_{v}\left(U_{1}\left(\xi_{\Delta}, t\right), V_{1}\left(\xi_{\Delta}, t\right)\right)=\epsilon^{-\frac{1}{2}} V_{0}^{\prime}\left(\xi_{\Delta}\right) d \Delta(t)+d R_{v}\left(V_{0}\left(\xi_{\Delta}\right), \xi, t\right)
\end{aligned}
$$

with $L_{u}, L_{v}$ non-self-adjoint linear operators

$$
\begin{aligned}
L_{u}\left(A_{1}, A_{2}\right)= & c \frac{d A_{1}}{d \xi}+h^{\prime}\left(U_{0}\right) A_{1}+Q_{u} \int w_{e}\left(\xi-\xi^{\prime}\right) f^{\prime}\left(U_{0}\left(\xi^{\prime}\right)\right) A_{1}\left(\xi^{\prime}\right) d \xi^{\prime} \\
& -Q_{v} \int w_{i}\left(\xi-\xi^{\prime}\right) f^{\prime}\left(V_{0}\left(\xi^{\prime}\right)\right) A_{2}\left(\xi^{\prime}\right) d \xi^{\prime} \\
L_{v}\left(A_{1}, A_{2}\right)= & c \frac{d A_{2}}{d \xi}+h^{\prime}\left(V_{0}\right) A_{2}+Q_{v} \int w_{e}\left(\xi-\xi^{\prime}\right) f^{\prime}\left(V_{0}\left(\xi^{\prime}\right)\right) A_{2}\left(\xi^{\prime}\right) d \xi^{\prime} \\
& -Q_{u} \int w_{i}\left(\xi-\xi^{\prime}\right) f^{\prime}\left(U_{0}\left(\xi^{\prime}\right)\right) A_{1}\left(\xi^{\prime}\right) d \xi^{\prime}
\end{aligned}
$$

for any functions $A_{1}(\xi), A_{2}(\xi) \in L_{2}(\mathbb{R})$. Finally, for all terms in equations (3.30) and (3.31) to be of the same order we require that $\Delta(t)=\mathcal{O}\left(\varepsilon^{1 / 2}\right)$. It then follows that $U_{0}(\xi-\Delta(t))=U_{0}(\xi)+\mathcal{O}\left(\varepsilon^{1 / 2}\right)$ etc., and equations (3.30) and (3.31) reduce to

$d U_{1}(\xi, t)-L_{u}\left(U_{1}(\xi, t), V_{1}(\xi, t)\right)=\epsilon^{-\frac{1}{2}} U_{0}^{\prime}(\xi) d \Delta(t)+d R_{u}\left(U_{0}(\xi), \xi, t\right)$

$d V_{1}(\xi, t)-L_{v}\left(U_{1}(\xi, t), V_{1}\left(\xi_{\Delta}, t\right)\right)=\epsilon^{-\frac{1}{2}} V_{0}^{\prime}(\xi) d \Delta(t)+d R_{v}\left(V_{0}(\xi), \xi, t\right)$

Let $\mathbf{L}$ denote the vector-valued operator with components $L_{u}, L_{v}$. That, is

$$
\mathbf{L}\left(\begin{array}{c}
A_{1} \\
A_{2}
\end{array}\right)=\left(\begin{array}{c}
L_{u}\left(A_{1}, A_{2}\right) \\
L_{v}\left(A_{1}, A_{2}\right) .
\end{array}\right)
$$

It can be shown that for a sigmoid firing rate function and Gaussian weight distributions, the operator $\mathbf{L}$ has a $1 \mathrm{D}$ null space spanned by $\left(U_{0}^{\prime}(\xi), V_{0}^{\prime}(\xi)\right)^{T}$ [33]. (The fact that $\left(U_{0}^{\prime}(\xi), V_{0}^{\prime}(\xi)\right)^{T}$ belongs to the null space follows immediately from differentiating equations (3.29) with respect to $\xi$ ). We then have the solvability condition for the existence of a nontrivial solution of equations (3.32) and (3.33), namely, that the inhomogeneous part is orthogonal to all elements of the null space of the adjoint operator $\mathbf{L}^{*}$. The latter is defined with respect to the inner product

$$
\int_{-\infty}^{\infty} \mathbf{B}(\xi) \cdot \mathbf{L} \mathbf{A}(\xi) d \xi=\int_{-\infty}^{\infty} \mathbf{L}^{*} \mathbf{B}(\xi) \cdot \mathbf{A}(\xi) d \xi
$$


where $\mathbf{A}(\xi)$ and $\mathbf{B}(\xi)$ are two-vectors with integrable components. We find that

$$
\mathbf{L}^{*}\left(\begin{array}{c}
B_{1} \\
B_{2}
\end{array}\right)=\left(\begin{array}{c}
L_{u}^{*}\left(B_{1}, B_{2}\right) \\
L_{v}^{*}\left(B_{1}, B_{2}\right),
\end{array}\right)
$$

where

$$
\begin{aligned}
L_{u}^{*}\left(B_{1}, B_{2}\right) & =-c_{\epsilon} \frac{d B_{1}}{d \xi}+h^{\prime}\left(U_{0}\right) B_{1}+f^{\prime}\left(U_{0}\right) Q_{u} \int w_{e}\left(\xi-\xi^{\prime}\right) B_{1}\left(\xi^{\prime}\right) d \xi^{\prime} \\
& -f^{\prime}\left(V_{0}\right) q_{v} \int w_{i}\left(\xi-\xi^{\prime}\right) B_{2}\left(\xi^{\prime}\right) d \xi^{\prime}
\end{aligned}
$$

and

$$
\begin{aligned}
L_{v}^{*}\left(B_{1}, B_{2}\right) & =-c_{\epsilon} \frac{d B_{2}}{d \xi}+h^{\prime}\left(V_{0}\right) B_{2}+f^{\prime}\left(V_{0}\right) Q_{v} \int w_{e}\left(\xi-\xi^{\prime}\right) B_{2}\left(\xi^{\prime}\right) d \xi^{\prime} \\
& -f^{\prime}\left(U_{0}\right) Q_{u} \int w_{i}\left(\xi-\xi^{\prime}\right) B_{1}\left(\xi^{\prime}\right) d \xi^{\prime}
\end{aligned}
$$

The adjoint operator $\mathbf{L}^{*}$ also has a one-dimensional null-space, that is, it is spanned by some vector-valued function $\mathcal{V}(\xi)$. Thus taking the inner product of both sides of equations (3.32) and (3.33) with respect to $\mathcal{V}(\xi)$ leads to the solvability condition

$$
\begin{aligned}
0= & \int_{-\infty}^{\infty} \mathcal{V}_{1}(\xi)\left[U_{0}^{\prime}(\xi) d \Delta(t)+\epsilon^{1 / 2} d R_{u}\left(U_{0}, \xi, t\right)\right] d \xi \\
& +\int_{-\infty}^{\infty} \mathcal{V}_{2}(\xi)\left[V_{0}^{\prime}(\xi) d \Delta(t)+\epsilon^{1 / 2} d R_{v}\left(V_{0}, \xi, t\right)\right] d \xi
\end{aligned}
$$

Thus $\Delta(t)$ satisfies the stochastic differential equation (SDE)

$$
d \Delta(t)=-\epsilon^{1 / 2} \frac{\int_{-\infty}^{\infty}\left(\mathcal{V}_{1}(\xi) d R_{u}\left(U_{0}, \xi, t\right)+\mathcal{V}_{2}(\xi) d R_{v}\left(V_{0}, \xi, t\right)\right) d \xi}{\int_{-\infty}^{\infty}\left(\mathcal{V}_{1}(\xi) U_{0}^{\prime}(\xi)+\mathcal{V}_{2}(\xi) V_{0}^{\prime}(\xi)\right) d \xi} .
$$

Using the lowest order approximations $d R_{u}\left(U_{0}, \xi, t\right)=g\left(U_{0}(\xi)\right) d W_{u}(\xi, t)$ and $d R_{v}\left(V_{0}, \xi, t\right)=g\left(V_{0}(\xi)\right) d W_{v}(\xi, t)$, we deduce that (for $\left.\Delta(0)=0\right)$

$$
\langle\Delta(t)\rangle=0, \quad\left\langle\Delta(t)^{2}\right\rangle=2 D(\varepsilon) t
$$

where $D(\epsilon)$ is the effective diffusivity

$$
D(\epsilon)=\epsilon \frac{\int_{-\infty}^{\infty}\left(\mathcal{V}_{1}(\xi)^{2} g\left(U_{0}(\xi)\right)^{2}+\mathcal{V}_{2}(\xi)^{2} g\left(V_{0}(\xi)\right)^{2}\right) d \xi}{\left[\int_{-\infty}^{\infty}\left(\mathcal{V}_{1}(\xi) U_{0}^{\prime}(\xi)+\mathcal{V}_{2}(\xi) V_{0}^{\prime}(\xi)\right) d \xi\right]^{2}}
$$

Note that since $\Delta(t)=\mathcal{O}\left(\epsilon^{1 / 2}\right)$, equation (3.26) implies that $U(x, t)=U_{0}(x-$ $\left.c_{\epsilon} t\right)+\mathcal{O}\left(\epsilon^{1 / 2}\right)$. Hence, averaging with respect to the noise shows that $\langle U(x, t)\rangle=$ $U_{0}\left(x-c_{\epsilon} t\right)+\mathcal{O}\left(\epsilon^{1 / 2}\right)$. A similar result holds for $V$. Thus, in the case of weak noise, averaging over many realizations of the stochastic wave generates a mean wave whose speed is approximately equal to $c_{\epsilon}$. This is indeed found to be the case numerically, see below. 


\subsection{Explicit results for a Heaviside rate function}

In order to illustrate the above analysis, we consider a particular example where the mean speed $c_{\epsilon}$ and diffusion coefficient $D(\epsilon)$ can be calculated explicitly. That is, set $g(U)=g_{0} U$ for the multiplicative noise term and take $f(U)=H(u-\kappa)$. (The constant $g_{0}$ has units of $\sqrt{\text { length/time })}$. The deterministic equations (3.28) and (3.29) for $\left(U_{0}, V_{0}\right)$ reduce to

$$
\begin{aligned}
& -c \frac{d U_{0}}{d \xi}+\gamma(\epsilon) U_{0}=Q_{u} \int_{-\infty}^{0} w_{e}\left(\xi-\xi^{\prime}\right) d \xi^{\prime}-Q_{v} \int_{X}^{\infty} w_{i}\left(\xi-\xi^{\prime}\right) d \xi^{\prime}+I \\
& -c \frac{d V_{0}}{d \xi}+\gamma(\epsilon) V_{0}=Q_{v} \int_{X}^{\infty} w_{e}\left(\xi-\xi^{\prime}\right) d \xi^{\prime}-Q_{u} \int_{-\infty}^{0} w_{i}\left(\xi-\xi^{\prime}\right) d \xi^{\prime}+I
\end{aligned}
$$

where

$$
\gamma(\epsilon)=1-\epsilon C(0) g_{0}^{2}
$$

These equations can be solved along identical lines to equations (3.8) and (3.9), and lead to the modified threshold conditions

$$
\kappa=\frac{1}{\gamma(\epsilon)} \int_{0}^{\infty} \mathrm{e}^{-s} \Psi_{X}(c s / \gamma(\epsilon)) d s+I, \quad \kappa=\frac{1}{\gamma(\epsilon)} \int_{0}^{\infty} \mathrm{e}^{-s} \Phi_{X}(-c s / \gamma(\epsilon)) d s+I .
$$

It immediately follows that both the speed $c$ and displacement $X$ depend on the noise strength $\epsilon$. The corresponding wave profiles are, see equations (3.15) and (3.16),

$$
U(\xi)=\frac{1}{c} \int_{0}^{\infty} \mathrm{e}^{-z \gamma / c} \Psi_{X}(z+\xi) d z+I
$$

and

$$
V(\xi)=\frac{1}{c} \int_{0}^{\infty} \mathrm{e}^{-z \gamma / c} \Phi_{X}(-z-\xi+X) d z+I .
$$

In order to calculate the diffusion coefficient, it is first necessary to determine the null vector $\mathcal{V}(\epsilon)$ of the adjoint linear operator $\mathbf{L}^{*}$. Substituting $f(U)=H(U-\kappa)$ and $g(U)=g_{0} U$ in equations (3.37) and (3.38) shows that the components of $\mathcal{V}$ satisfy the simultaneous equations

$$
\begin{aligned}
& c \frac{d \mathcal{V}_{1}}{d \xi}+\gamma(\epsilon) \mathcal{V}_{1}=\frac{\delta(\xi)}{\left|U_{0}^{\prime}(0)\right|} Q_{u} \int w_{e}(z) \mathcal{V}_{1}(z) d z-\frac{\delta(\xi-X)}{\left|V_{0}^{\prime}(X)\right|} Q_{v} \int w_{i}(z-X) \mathcal{V}_{2}(z) d z \\
& c \frac{d \mathcal{V}_{2}}{d \xi}+\gamma(\epsilon) \mathcal{V}_{2}=-\frac{\delta(\xi)}{\left|U_{0}^{\prime}(0)\right|} Q_{u} \int w_{i}(z) \mathcal{V}_{1}(z) d z+\frac{\delta(\xi-X)}{\left|V_{0}^{\prime}(X)\right|} Q_{v} \int w_{e}(z-X) \mathcal{V}_{2}(z) d z
\end{aligned}
$$

Proceeding along similar lines to $[19,49]$, we make the ansatz that

$$
\begin{aligned}
& \mathcal{V}_{1}(\xi)=\mathcal{A}_{1} \mathrm{e}^{-\gamma \xi / c} H(\xi)-\mathcal{B}_{1} \mathrm{e}^{-\gamma[\xi-X] / c} H(\xi-X), \\
& \mathcal{V}_{2}(\xi)=-\mathcal{A}_{2} \mathrm{e}^{-\gamma \xi / c} H(\xi)+\mathcal{B}_{2} \mathrm{e}^{-\gamma[\xi-X] / c} H(\xi-X) .
\end{aligned}
$$

Substituting back into the adjoint equations yields algebraic conditions for the constant coefficients $\mathcal{A}_{j}, \mathcal{B}_{j}$ :

$$
\begin{aligned}
& \mathcal{A}_{1}=\frac{Q_{u}}{\left|U_{0}^{\prime}(0)\right|}\left(\mathcal{A}_{1} \Omega_{e}[0]-\mathcal{B}_{1} \Omega_{e}[X]\right) \\
& \mathcal{B}_{1}=\frac{Q_{v}}{\left|V_{0}^{\prime}(X)\right|}\left(\mathcal{B}_{2} \Omega_{i}[0]-\mathcal{A}_{2} \Omega_{i}[-X]\right)
\end{aligned}
$$


Stochastic neural field model of binocular rivalry waves

$$
\begin{aligned}
& \mathcal{A}_{2}=\frac{Q_{u}}{\left|U_{0}^{\prime}(0)\right|}\left(\mathcal{A}_{1} \Omega_{i}[0]-\mathcal{B}_{1} \Omega_{i}[X]\right) \\
& \mathcal{B}_{2}=\frac{Q_{v}}{\left|V_{0}^{\prime}(X)\right|}\left(\mathcal{B}_{2} \Omega_{e}[0]-\mathcal{A}_{2} \Omega_{e}[-X]\right),
\end{aligned}
$$

where

$$
\Omega_{j}[x]=\int_{0}^{\infty} \mathrm{e}^{-z \gamma / c} w_{j}(x+z) d z, \quad j=e, i .
$$

Differentiating equations (3.47) and (3.48) with respect to $\xi$ and using equations (3.12) and (3.13), shows that

$$
U_{0}^{\prime}(0)=-Q_{u} \Omega_{e}[0]-Q_{v} \Omega_{i}[-X]<0,
$$

and

$$
V_{0}^{\prime}(X)=Q_{v} \Omega_{e}[0]+Q_{u} \Omega_{i}[X]>0 .
$$

We have also used the fact that the weight distributions $w_{e}(x), w_{i}(x)$ are even functions of $x$. Substituting these derivatives into equations (3.52) and (3.55) gives

$$
\mathcal{B}_{2}=-\frac{Q_{v} \Omega_{e}[-X]}{Q_{u} \Omega_{i}[X]} \mathcal{A}_{2}, \quad \mathcal{B}_{1}=-\frac{Q_{v} \Omega_{i}[-X]}{Q_{u} \Omega_{e}[X]} \mathcal{A}_{1} .
$$

It follows from equations (3.54) and (3.59) that $\mathcal{A}_{2}, \mathcal{B}_{1}, \mathcal{B}_{2}$ can all be expressed as constant multiples of $\mathcal{A}_{1}$, with the latter determined by normalizing the null vector.

\section{Numerical results}

In order to proceed analytically, it was convenient to make an adiabatic approximation where we assumed that the depression variables $q_{u}$ and $q_{v}$ do not change with time. We also made the further approximation that the fronts move as a composite wave and never drift with respect to each other. In this section we verify that our analytical assumptions are reasonable by considering numerical simulations of the full system given by equations (3.17), (3.18), (3.2) and (3.4) with $Q_{u, v} \rightarrow q_{u, v}$, though we retain the Heaviside firing rate function to allow us to compare directly the simulations and the analysis. Simulations were performed on a constant lattice spaced grid of length $\Delta x$ with constant time step $\Delta t$ such that the size of the composite waves is far smaller than the simulation region. Fig. 10 shows that our predicted mean front shape develops into a stochastic traveling wave moving at the speed resulting from our analysis. It can be seen that there is very good agreement between the mean profile and stochastic wavefront position. Moreover, given that the wave speed is approximately constant helps to verify our assumption that $q_{u}$ and $q_{v}$ do not change significantly enough to affect the wave motion.

In Fig. 11 we plot the mean and variance of wave position as a function of time, starting from initial conditions which are solutions to equations (3.28) and (3.29). In order to numerically calculate the mean location of the front as a function of time, we carry out a large number of level set position measurements of the front associated with the left eye, say. That is, we determine the positions $X_{a}(t)$ such that $U\left(X_{a}(t), t\right)=a$, for various level set values $a \in(0.5 \kappa, 1.3 \kappa)$ and then define the mean location to be $\bar{X}(t)=\mathbb{E}\left[X_{a}(t)\right]$, where the expectation is first taken with respect to the sampled values $a$ and then averaged over $N$ trials. The corresponding variance is given by $\sigma_{X}^{2}(t)=\mathbb{E}\left[\left(X_{a}(t)-\bar{X}(t)\right)^{2}\right]$. It can be seen that $\bar{X}(t)$ varies linearly with $t$, consistent with the assumption that there is constat speed wave, $\bar{X}(t) \sim c_{\varepsilon} t$. The 

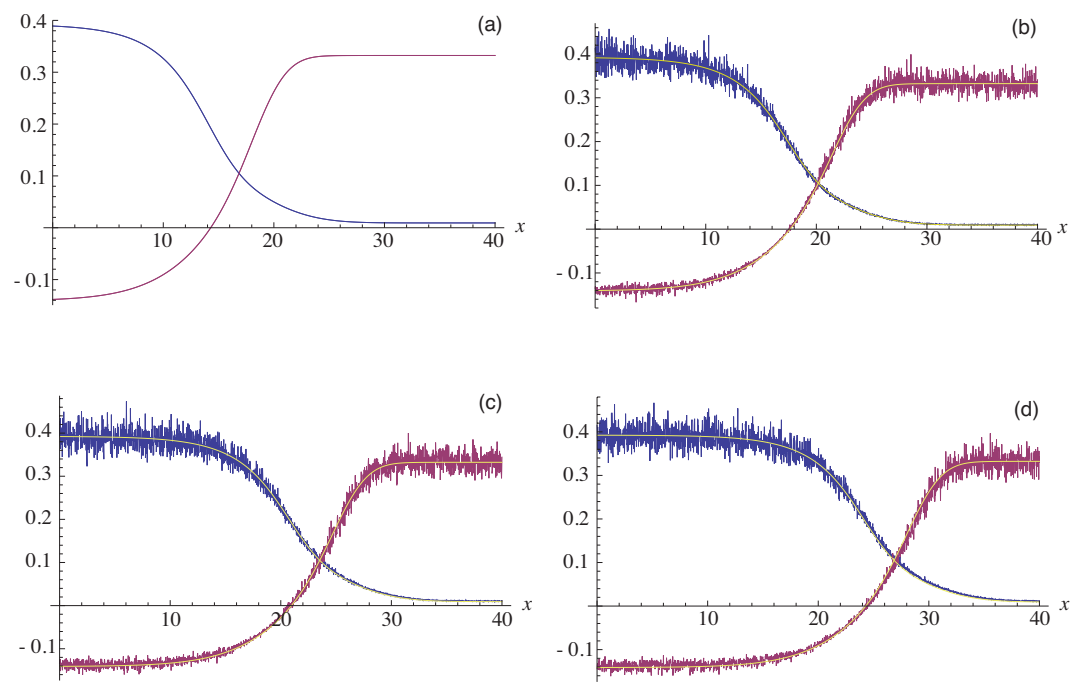

Figure 10. Effects of multiplicative noise in the activity variables. Simulation of the activity variables $u(x, t)$ (blue) and $v(x, t)$ (purple) for (a) $\mathrm{t}=0$, (b) $\mathrm{t}=1$, (c) $\mathrm{t}=2$, (d) $\mathrm{t}=3$. The continuous lines are the functions $U_{0}(x-c t)$ and $V_{0}(x-c t)$ which were found by solving equation (3.29). The functions $U_{0}$ and $V_{0}$ were also used for the initial condition. Parameter values were $a_{i}=1, a_{e}=0.4, \sigma_{i}=1, \sigma_{e}=$ $2, \beta=5, \kappa=0.05, I=0.24, \epsilon=0.006$ with spatial and temporal grid sizes both being 0.01 .
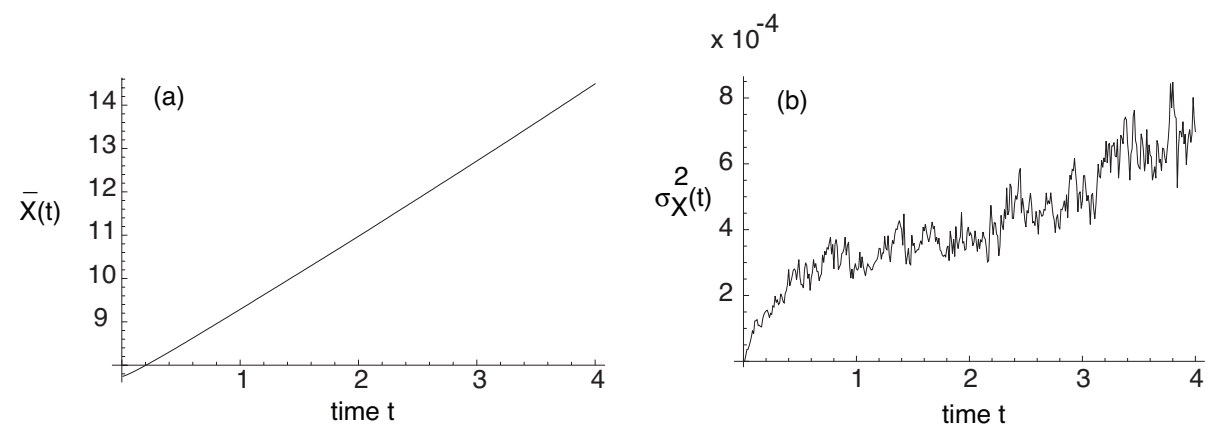

Figure 11. (a) Mean and (b) variance of the wave position for an ensemble of 128 stochastic simulations. Parameters were the same as Fig. 10

variance initially increases rapidly with respect to $t$, but then follows linear behaviour consistent with a diffusive-like displacement of the wave from its uniformly translating position at long time scales, $\sigma_{X}^{2}(t) \sim 2 D(\varepsilon) t$. The initial sharp increase in the variance results from the fact that the left and right fronts do move slightly with respect to each other, resulting in higher order behaviour. However, we find numerically that this component of the variance is small and bounded so that it becomes negligible as time increases. In order to show this, we carry out corresponding level set measurements of the right- front position by introducing $Y_{a}(t)$ such that $V\left(Y_{a}(t), t\right)=a$. We then define the difference in front positions according to $\Delta X_{a}(t)=X_{a}(t)-Y_{a}(t)$ and set $\Delta X(t)=\mathbb{E}\left[X_{a}(t)-Y_{a}(t)\right]$. Fig 12 shows a time plot of $\Delta X(t)$ for a fully developed 


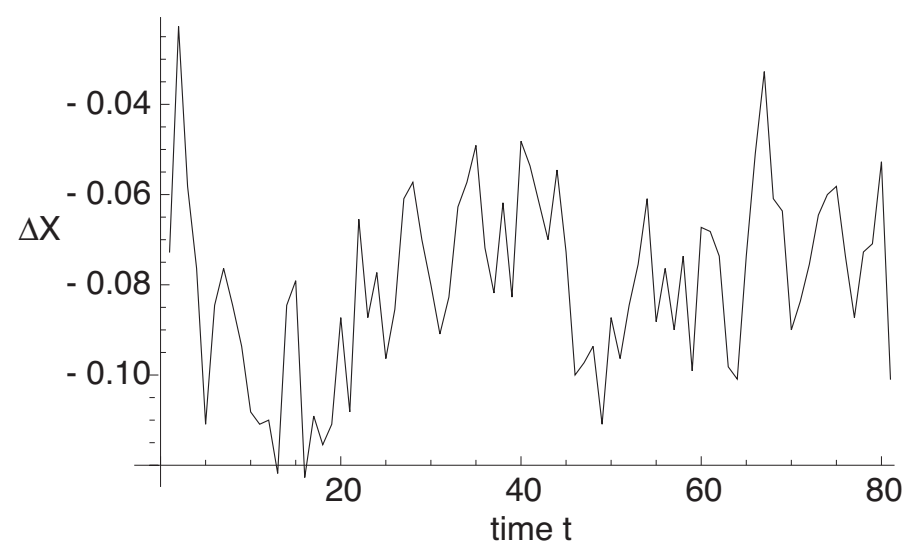

Figure 12. Difference $\Delta X$ in the position of the left-eye and right-eye fronts as a function of time $t$.

stochastic wave, which verifies that this difference in motion is small (same order as the grid spacing for this particular simulation) and bounded.

Since our analysis predicts that the wave position will follow a Brownian motion, the time taken for a wave to travel a distance $L>0$ has a distribution given by the standard first passage time formula for Brownian motion with drift $c$. That is, let $T_{L}$ denote the first passage time for the wave to travel a distance $L: c T_{L}+\Delta\left(T_{L}\right)=L$ given $\Delta(0)=0$. Then the first passage time density is given by an inverse Gaussian or Wald distribution:

$$
f\left(T_{L}\right)=\mathcal{F}\left(T_{L} ; \frac{L}{c}, \frac{L^{2}}{D}\right),
$$

where

$$
\mathcal{F}(T ; \mu, \lambda)=\left[\frac{\lambda}{2 \pi T^{3}}\right]^{1 / 2} \exp \left(-\frac{\lambda(T-\mu)^{2}}{2 \mu^{2} T}\right) .
$$
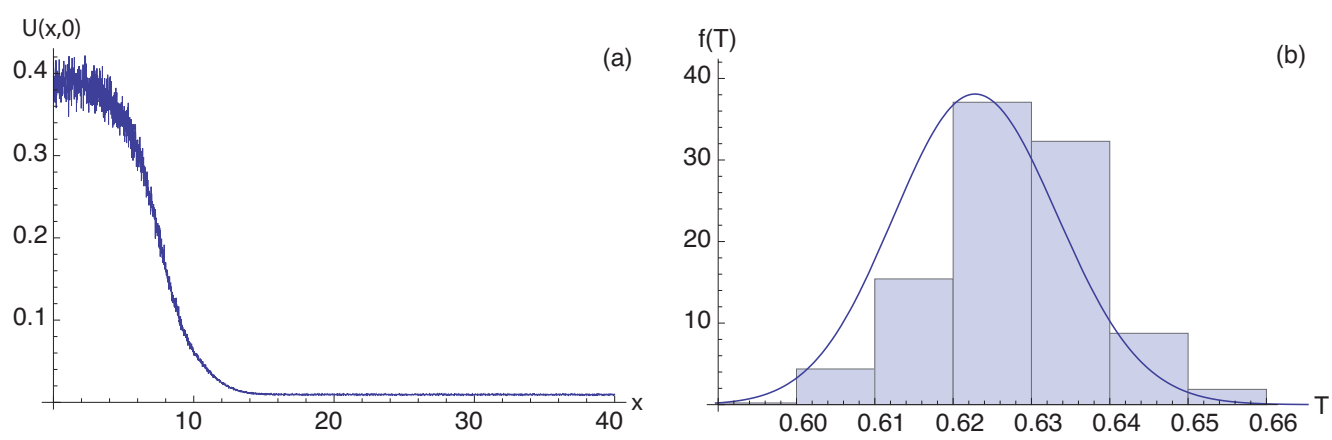

Figure 13. (a) Snapshot of a stochastic traveling wave (only left eye activity is shown). (b) First passage time distribution for the wave to travel a distance $L=1$, starting from the wave profile in (a). Parameter values were $a_{i}=1, a_{e}=0.4, \sigma_{i}=$ $1, \sigma_{e}=2, \beta=5, \kappa=0.05, I=0.24, \epsilon=0.006$ with spatial and temporal grid sizes both being 0.01 . The best fit inverse Gaussian $\mathcal{F}(T ; \mu, \lambda)$ for the histogram gives the parameters $\mu=0.62, \lambda=2200$ which is in very good agreement with the theoretical predictions of $\mu=L / c=0.6, \lambda=L^{2} / D=2100$. 

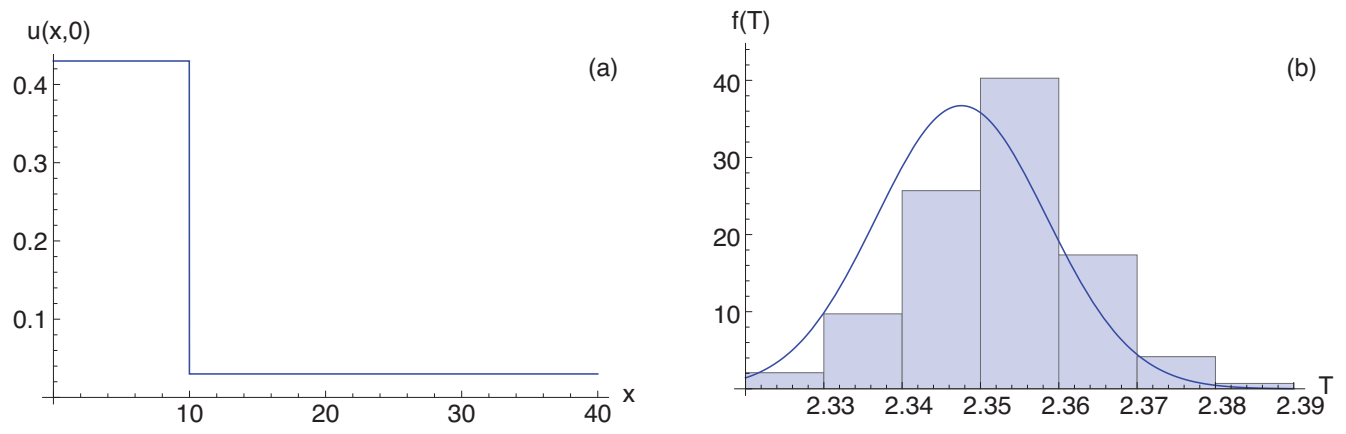

Figure 14. Same as Fig. 13 except that the initial condition is taken to be a righteye dominant homogeneous steady-state perturbed by a step-function in left-eye activity. Although the wave now needs time to develop into a stochastic traveling front, the best fit inverse Gaussian still shows strong qualitative agreement.
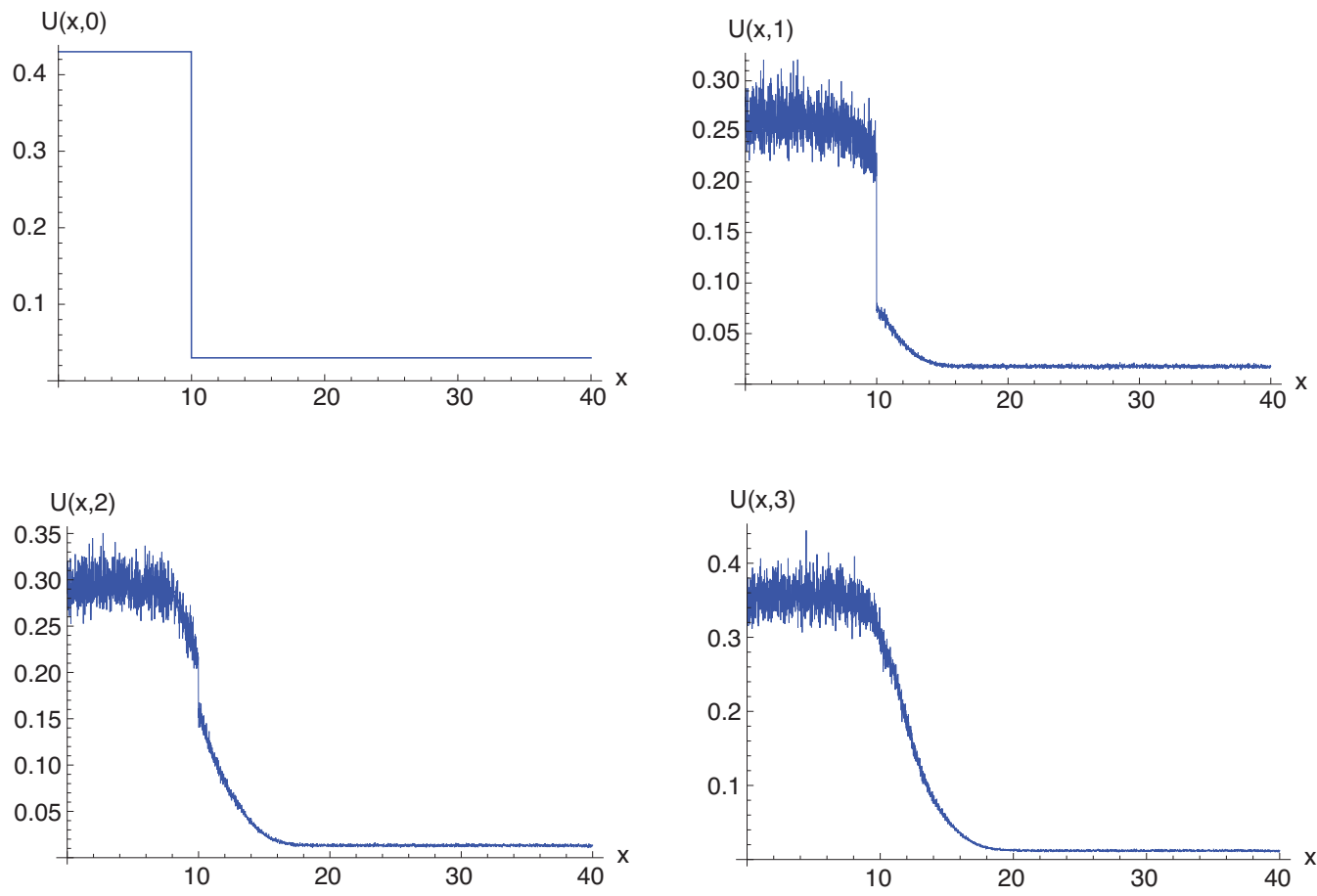

Figure 15. Simulation of the activity variable $U(x, t)$ evolving from a step input in the left-eye activity for the times $t=0,1,2,3$ with the same parameters as in Fig. 13.

Fig. 13 shows the first passage time distribution generated over a large number of simulations for an initial condition given by a fully developed stochastic traveling wave. We find good agreement with our analysis, namely, that the distribution can be fitted by the predicted inverse Gaussian. If we choose to consider more experimentally realistic initial conditions, which represent a sudden change of contrast in a small region of the depressed stimulus, we find that the wave takes a substantial time to 
develop, which results in a slightly modified first passage distribution, athough it is still well-fitted by an inverse Gaussian, as can be seen in Fig. 14. The corresponding development of the wave is illustrated in Fig. 15.

So far we have assumed that all the noise is in the activity variables. We now study numerically how noise affects first passage time distributions when placed in the depression variables. We assume once again that a traveling wave crosses cortex significantly faster than the relaxation time of synaptic depression, so that $q_{u}$ and $q_{v}$ can be taken to be constant with respect to time. However, they are no longer constant with respect to space nor with respect to trials, that is, $q_{u}=Q_{u}(x)$ and $q_{v}=Q_{v}(x)$ with $Q_{u}, Q_{v}$ random variables of $x$. So although the wave itself will travel deterministically in a given trial, the functions $Q_{u}(x)$ and $Q_{v}(x)$ will be different across trials. In several experimental studies $[46,47]$ of binocular rivalry waves, a dominance switch was induced about half-way through a normal dominance cycle by locally changing the contrast in the depressed stimulus. The latter can be represented by increasing the input strength $I$ over a small region in our model. This suggests taking $q_{u}$ and $q_{v}$ to evolve according to

$$
\begin{aligned}
& \tau_{s} d q_{u}(x, t)=\left[1-q_{u}(x, t)\right] d t+\eta d W_{q_{u}}(x, t) \\
& \tau_{s} d q_{v}(x, t)=\left[1-q_{v}-\beta q_{v}\right] d t+\eta d W_{q_{v}}(x, t)
\end{aligned}
$$

over the time interval $\left[t_{0}, T\right]$. It is assumed that a switch from left to right eye dominance occurs at $t=t_{0}$ so that $u(x, t)<\kappa$ and $v(x, t)>\kappa$ for $t \in\left(t_{0}, T\right)$. The time $T$ is then chosen so that the system is about $2 / 3$ of the way through a rivalry oscillation, such that $Q_{u}(x)=q_{u}(x, T)$ and $Q_{v}(x)=q_{v}(x, T)$. (Similar results were obtained for different cycle lengths and choices of $T$ away from the beginning or end of a cycle). Thus, the quenched random variables $Q_{u}(x)$ and $Q_{v}(x)$ are obtained by taking a snapshot of two lines of independent Ornstein-Uhlenbeck processes. Figs. 16 and 17 show numerically that the first passage time distribution is still well approximated by an inverse Gaussian distribution. Fig. 18 illustrates the development and propagation of a wave on top of quenched random depression variables. As can be seen there is still an approximately constant overall shape, though the front's position will no longer move at a constant speed.

\section{Discussion}

In this paper we have analysed the effects of noise on a neural field model of binocular rivalry waves. Formulating the problem in terms of continuum neural field equations allowed us to study the short time behavior associated with the propagation of eye dominance from an analytical perspective, both for deterministic and stochastic waves. In the deterministic case, we have previously shown that some form of slow adaptation such as synaptic depression is needed in order to provide a symmetry breaking mechanism that allows propagation of a binocular rivalry wave [17]. That is, the equations for the left and right eye networks have to be different on the time scales during which traveling waves propagate. One particularly interesting implication of our previous work was that purely noise-driven switching between rivalrous states in the absence of adaptation couldn't by itself generate rivalry waves, since it would not produce bias in a particular direction. However, as we have shown in this paper, noise does have a significant effect on binocular rivalry waves in the presence of slow adaptation. In particular, our analysis predicts that motion of the wave in the 

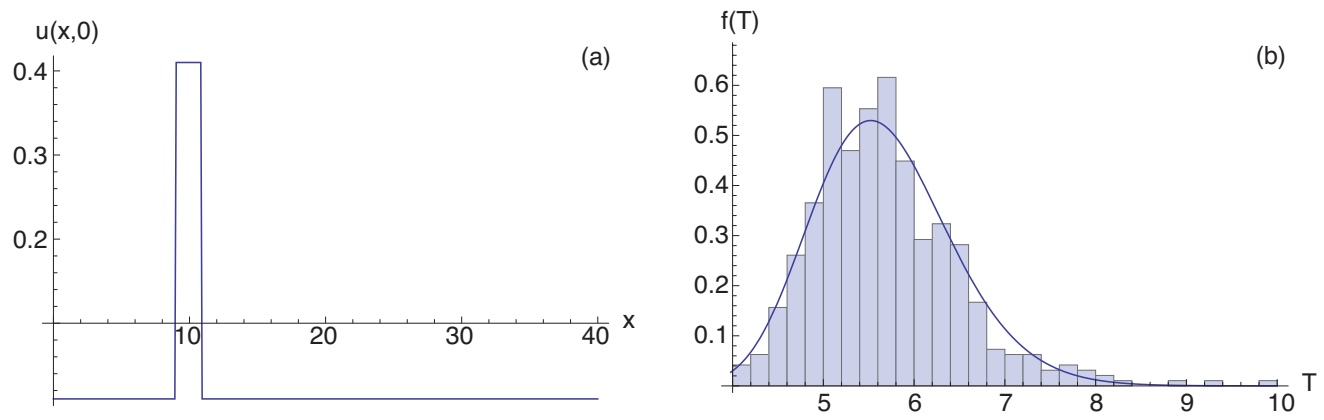

Figure 16. Effects of quenched noise in the depression variables. (a) Initial condition given by a right-eye dominant homogeneous steady state perturbed by a narrow square pulse of left-eye actiivity. (b) First passage time distribution for a wave to travel a distance $L=6$ starting from the initial condition shown in (a). Parameter values were $a_{i}=1, a_{e}=0.4, \sigma_{i}=1, \sigma_{e}=2, \beta=5, \kappa=0.05, I=$ $0.24, \eta=0.037\langle Q\rangle$, where $\langle Q\rangle$ is the spatial average of the quenched depression variables (to make the results comparable with multiplicative noise). The spatial grid size $\Delta x=0.1$ and the temporal grid step is $\Delta t=0.01$. The solid curve in (b) is the best fit inverse Gaussian.
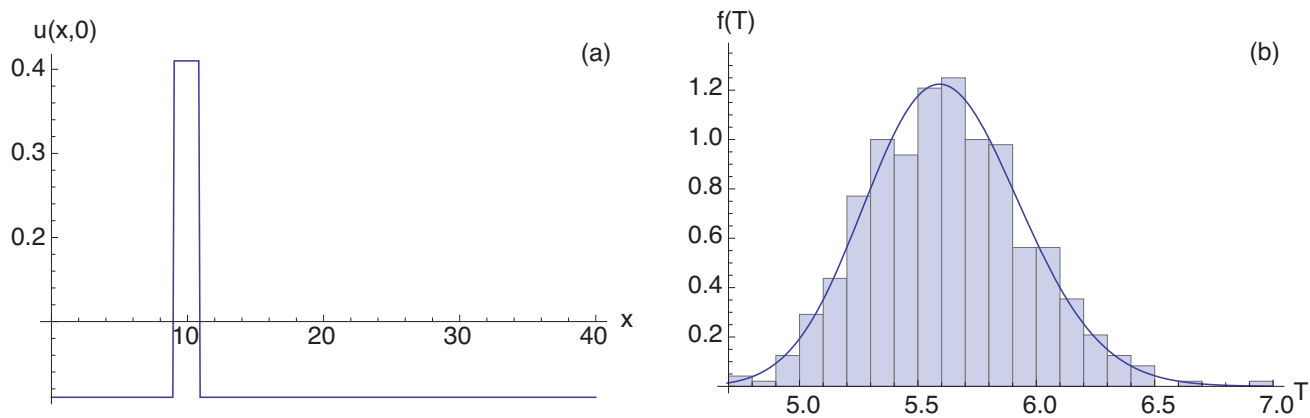

Figure 17. Same as in Fig.16, except the noise is now smaller with $\eta=0.025\langle Q\rangle$.

prsence of noise in the activity variables has a diffusive component that leads to a distribution of first passage times given by an inverse Gaussian. Such a result could be experimentally testable. Interestingly, we also found a similar distribution of first passage times for quenched noise in the depression variables.

In future work it would be interesting to extend our neural field model to a more realistic network topology. Instead of considering one line of neurons for each eye, we could consider a circle of neurons for each point in the visual field - this would allow us to take orientation information into account using an extension of the coupled ring model $[20,21]$. We could then investigate the experimental observation that the speed of binocular rivalry waves depends on the orientation of the left and right eye grating stimuli [85]. Moreover, experiments on waves crossing gaps in perceptual grating stimuli have found that gap crossing is a stochastic variable and we would hope that it is possible to extend our neural field framework to situations involving non-constant underlying orientations such as this one. 

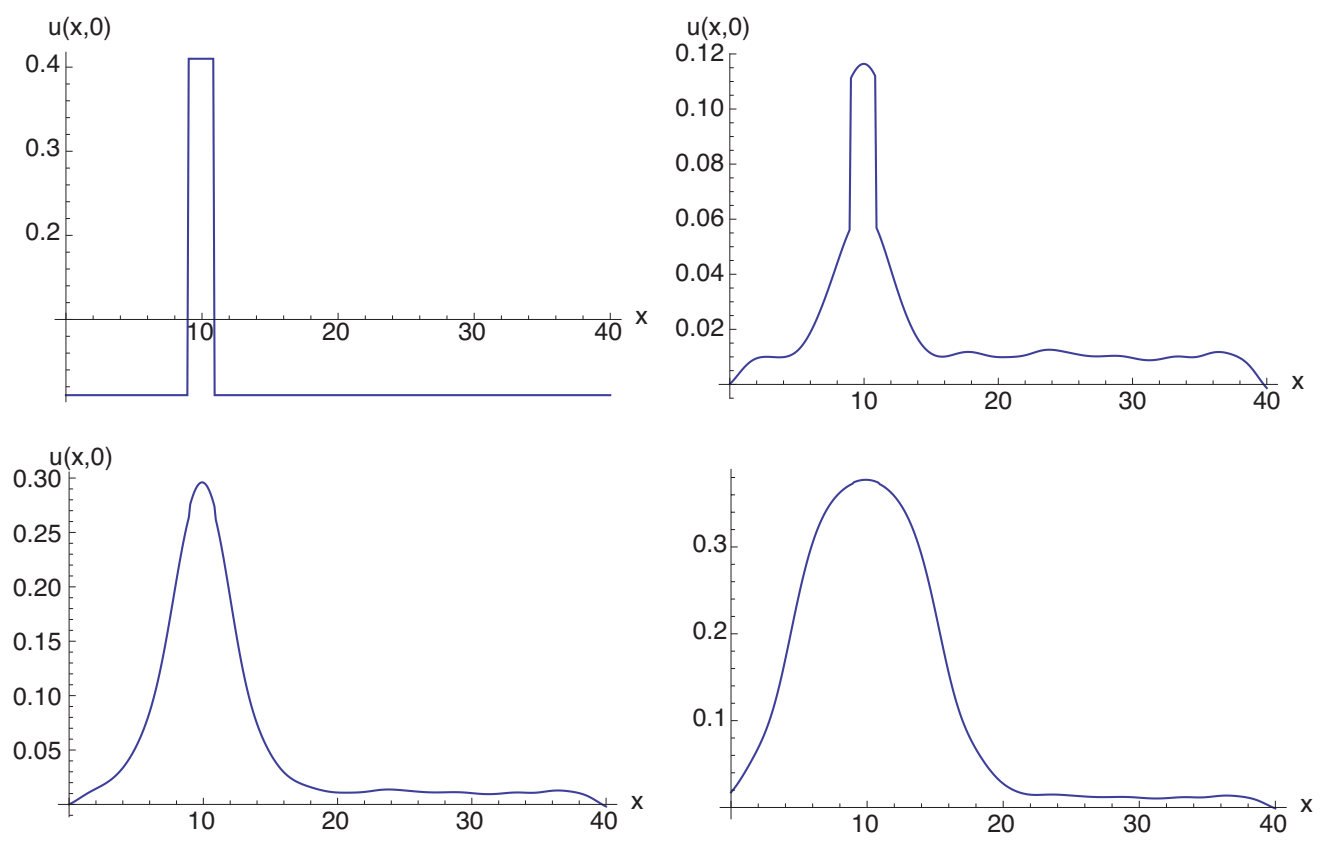

Figure 18. Development of an activity variable wave on top of quenched stochastic depression variables $Q_{u}(x), Q_{v}(x)$. Paramaters are the same as Fig. 17

\section{Acknowledgements}

This publication was based on work supported in part by the National Science Foundation (DMS-1120327), the King Abdullah University of Science and Technology Award No. KUK-C1-013-04, and the Systems Biology Doctoral Training Centre, University of Oxford.

\section{References}

[1] L Schimansky-Geier A S Mikhailov and W Ebeling. Stochastic motion of the propagating front in bistable media. Phys. Rev. Lett., 96:453, 1983.

[2] S. Amari. Dynamics of pattern formation in lateral-inhibition type neural fields. Biological Cybernetics, 27(2):77-87, 1977.

[3] A. Angelucci, J.B. Levitt, E.J.S. Walton, J.M. Hupé, J. Bullier, and J.S. Lund. Circuits for local and global signal integration in primary visual cortex. The Journal of Neuroscience, 22(19):8633, 2002.

[4] J Armero, J Casademunt, L Ramirez-Piscina, and J M Sancho. Ballistic and diffusive corrections to front propagation in the presence of multiplicative noise. Phys. Rev. E, 58:5494-5500, 1998.

[5] E. Bart, S. Bao, and D. Holcman. Modeling the spontaneous activity of the auditory cortex. Journal of Computational Neuroscience, 19(3):357-378, 2005.

[6] R. Ben-Yishai, R. Lev Bar-Or, and H. Sompolinsky. Theory of orientation tuning in visual cortex. Proc. Nat. Acad. Sci., 92:3844-3848, 1995.

[7] R. Ben-Yishai, R.L. Bar-Or, and H. Sompolinsky. Theory of orientation tuning in visual cortex. Proceedings of the National Academy of Sciences, 92(9):3844, 1995.

[8] R. Blake. A primer on binocular rivalry, including current controversies. Brain and Mind, $2(1): 5-38,2001$.

[9] R. Blake and N.K. Logothetis. Visual competition. Nature Reviews Neuroscience, 3(1):13-21, 2002. 
[10] G G Blasdel and G Salama. Voltage-sensitive dyes reveal a modular organization in monkey striate cortex. Nature, 321:579-585, 1986.

[11] G.G. Blasdel. Orientation selectivity, preference, and continuity in monkey striate cortex. The Journal of Neuroscience, 12(8):3139, 1992.

[12] T Bonhoeffer and A Grinvald. Orientation columns in cat are organized in pinwheel like patterns. Nature, 364:166-146, 1991.

[13] C A Brackley and M S Turner. Random fluctuations of the firing rate function in a continuum neural field model. Phys. Rev. E, 75:041913, 2007.

[14] J. Braun and M. Mattia. Attractors and noise: Twin drivers of decisions and multistability. Neuroimage, 52(3):740-751, 2010.

[15] P C Bressloff. Stochastic neural field theory and the system-size expansion. SIAM J. Appl. Math, 70:1488-1521, 2009.

[16] P C Bressloff. Metastable states and quasicycles in a stochastic wilson-cowan model of neuronal population dynamics. Phys. Rev. E, 85:051903, 2010.

[17] P. C. Bressloff. Spatiotemporal dynamics of continuum neural fileds. J. Phys. A, 45:033001, 2012.

[18] P. C. Bressloff and M. A. Webber. Front propagation in stochastic neural fields. SIAM J. Appl. Dyn. Syst., page In press, 2012.

[19] P.C. Bressloff. Traveling fronts and wave propagation failure in an inhomogeneous neural network. Physica D: Nonlinear Phenomena, 155(1-2):83-100, 2001.

[20] P.C. Bressloff and J.D. Cowan. An amplitude equation approach to contextual effects in visual cortex. Neural computation, 14(3):493-525, 2002.

[21] P.C. Bressloff, J.D. Cowan, M. Golubitsky, P.J. Thomas, and M.C. Wiener. Geometric visual hallucinations, euclidean symmetry and the functional architecture of striate cortex. Philosophical Transactions of the Royal Society of London. Series B: Biological Sciences, 356(1407):299, 2001.

[22] P.C. Bressloff and M.A. Webber. Neural field model of binocular rivalry waves. Journal of Computational Neuroscience, 32:233-252, 2012.

[23] M Buice and J D Cowan. Field-theoretic approach to fluctuation effects in neural networks. Phys. Rev. E, 75:051919, 2007.

[24] M Buice, J D Cowan, and C C Chow. Systematic fluctuation expansion for neural network activity equations. Neural Comp., 22:377-426, 2010.

[25] M Camperi and X-J Wang. A model of visuospatial short-term memory in prefrontal cortex: recurrent network and cellular bistability. J. Comp. Neurosci., 5:383-405, 1998.

[26] D. Carmel, M. Arcaro, S. kastner, and U. Hasson. How to create and use binocular rivalry. J. Vis. Exp., 45:1-8, 2010.

[27] F.S. Chance, S.B. Nelson, and L.F. Abbott. Synaptic depression and the temporal response characteristics of v1 cells. The Journal of neuroscience, 18(12):4785, 1998.

[28] S Coombes. Waves, bumps and patterns in neural field theories. Biol. Cybern., 93:91-108, 2005.

[29] S. Coombes and M. Owen. Evans functions for integral neural field equations with heaviside firing rate function. Differential and Integral Equations, 16:513-536, 2003.

[30] S. Coombes, N. A. Venkov, I. Bojak, D. T. J. Liley, and C. R. Laing. Modeling electrocortical activity through improved local approximations of integral neural field equations. Phys. Rev. E, 76:051901, 2007.

[31] G B Ermentrout. Neural networks as spatio-temporal pattern-forming systems. Rep. Prog. Phy., 61:353-430, 1998.

[32] G B Ermentrout and J Cowan. A mathematical theory of visual hallucination patterns. Bio. Cybern., 34:137-150, 1979.

[33] G B Ermentrout and J B McLeod. Existence and uniqueness of travelling waves for a neural network. Proc. Roy. Soc. Edin. A, 123:461-478, 1993.

[34] J Gorecki F. de Pasquale and J Poielawski. On the stochastic correlations in a randomly perturbed chemical front. J. Phys. Chem., 25:433, 1992.

[35] O Faugeras, J Touboul, and B Cessac. A constructive mean-field analysis of multi-population neural networks with random synaptic weights and stochastic inputs. Frontiers in Comp. Neurosci., 3:1-28, 2009.

[36] D. Ferster and K.D. Miller. Neural mechanisms of orientation selectivity in the visual cortex. Annual review of neuroscience, 23(1):441-471, 2000.

[37] R. Fox and F. Rasche. Binocular rivalry and reciprocal inhibition. Attention, Perception, 85 Psychophysics, 5(4):215-217, 1969.

[38] A. W. Freeman. Multistage model for binocular rivalry. J. Neurophysiol., 94:4412-4420, 2005.

[39] C W Gardiner. Handbook of stochastic methods, 4th edition. Springer, Berlin, 2009. 
[40] M A Geise. Neural field theory for motion perception. Kluwer Academic Publishers, Dordrecht, 1999.

[41] C D Gilbert. Horizontal integration and cortical dynamics. Neuron, 9:1-13, 1992.

[42] X Huang, W C Troy, Q Yang, H Ma, C R Laing, S J Schiff, and J Wu. Spiral waves in disinhibited mammalian neocortex. J Neurosci, 24:9897-9902, 2004.

[43] D H Hubel and T N Wiesel. Sequence regularity and geometry of orientation columns in the monkey striate cortex. J. Comp. Neurol., 158:267-294, 1974.

[44] D H Hubel and T N Wiesel. Uniformity of monkey striate cortex: A parallel relationship between field size, scatter, and magnification factor. J. Comp. Neurol., 158:295-306, 1974.

[45] A Hutt, A Longtin, and L Schimansky-Geier. Additive noise-induces turing transitions in spatial systems with application to neural fields and the swift-hohenberg equation. Physica $D, 237: 755-773,2008$.

[46] M.S. Kang, D. Heeger, and R. Blake. Periodic perturbations producing phase-locked fluctuations in visual perception. Journal of vision, 9(2), 2009.

[47] M.S. Kang, S.H. Lee, J. Kim, D. Heeger, and R. Blake. Modulation of spatiotemporal dynamics of binocular rivalry by collinear facilitation and pattern-dependent adaptation. Journal of vision, 10(11), 2010.

[48] J P Keener. Waves in excitable media. SIAM J. Appl. Math., 39:528-548, 1981.

[49] Z P Kilpatrick, S E Folias, and P C Bressloff. Traveling pulses and wave propagation failure in inhomogeneous neural media. SIAM J. Appl. Dyn. Syst., 7:161-185, 2008.

[50] Z.P. Kilpatrick and P.C. Bressloff. Binocular rivalry in a competitive neural network with synaptic depression. SIAM Journal on Applied Dynamical Systems, 9:1303-1347, 2010.

[51] Y Kuramoto. Chemical Oscillations, Waves and Turbulence. Springer-Verlag, New-York, 1984.

[52] C R Laing and W C Troy. Pde methods for nonlocal models. SIAM J. Appl. Dyn. Syst., $2: 487-516,2003$.

[53] C R Laing, W C Troy, B Gutkin, and G B Ermentrout. Multiple bumps in a neuronal model of working memory. SIAM J. Appl. Math, 63:62-97, 2002.

[54] C.R. Laing and C.C. Chow. A spiking neuron model for binocular rivalry. Journal of computational neuroscience, 12(1):39-53, 2002.

[55] S.H. Lee, R. Blake, and D.J. Heeger. Travelling waves of activity in primary visual cortex during binocular rivalry. Nature neuroscience, 8(1):22, 2005.

[56] S. R. Lehky. An astable multivibrator model of binocular rivalry. Perception, 17:215-228, 1988.

[57] S. R. Lehky. Binocular rivalry is not chaotic. Proc Roy Soc Lond: Biol Sci., 259:71-76, 1995.

[58] S LeVay and S B Nelson. Columnar organization of the visual cortex. In A G Leventhal, editor, The neural basis of visual function, pages 266-315. CRC Press, Boca Raton, Fl, 1991.

[59] D J T Liley, P J Cadusch, and M P Dafilis. A spatially continuous mean field theory of electrocortical activity. Network, 13:67-113, 2002.

[60] N. K. Logothetis, D. A. Leopold, and D. L. Sheinberg. What is rivalling during binocular rivalry? Nature, 380:621-624, 1996.

[61] PN Loxley and PA Robinson. Soliton model of competitive neural dynamics during binocular rivalry. Physical review letters, 102(25):258701, 2009.

[62] R. Moreno-Bote, J. Rinzel, and N. Rubin. Noise-induced alternations in an attractor network model of perceptual bistability. Journal of Neurophysiology, 98(3):1125, 2007.

[63] E A Novikov. Functionals and the random-force method in turbulence theory. Sov. Phys. JETP, 20:1290, 1965.

[64] P I Nunez. Neocortical dynamics and human EEG rhythms. Oxford University Press, NY, 1995.

[65] K Obermayer and G G Blasdel. Geometry of orientation and ocular dominance columns in monkey striate cortex. J. Neurosci., 13:4114-4129, 1993.

[66] D.J. Pinto and G.B. Ermentrout. Spatially structured activity in synaptically coupled neuronal networks: I. traveling fronts and pulses. SIAM journal on Applied Mathematics, pages 206$225,2001$.

[67] K A Richardson, S J Schiff, and B J Gluckman. Control of traveling waves in the mammalian cortex. Phys. Rev. Lett., 94:028103, 2005.

[68] P A Robinson, C J Rennie, J J Wright, H Bahramali, E Gordon, and D I Rowe. Prediction of electroencephalographic spectra from neurophysiology. Phys Rev E, 63:021903, 2001.

[69] F Sagues, J M Sancho, and J Garcia-Ojalvo. Spatiotemporal order out of noise. Rev. Mod. Phys., 79:829-882, 2007.

[70] E Schwartz. Spatial mapping in the primate sensory projection: analytic structure and relevance to projection. Biol. Cybern., 25:181-194, 1977.

[71] A. Shpiro, R. Curtu, J. Rinzel, and N. Rubin. Dynamical characteristics common to neuronal 
competition models. Journal of neurophysiology, 97(1):462, 2007.

[72] A. Shpiro, R. Moreno-Bote, N. Rubin, and J. Rinzel. Balance between noise and adaptation in competition models of perceptual bistability. Journal of computational neuroscience, $27(1): 37-54,2009$.

[73] L.C. Sincich and G.G. Blasdel. Oriented axon projections in primary visual cortex of the monkey. The Journal of Neuroscience, 21(12):4416, 2001.

[74] F.K. Skinner, N. Kopell, and E. Marder. Mechanisms for oscillation and frequency control in reciprocally inhibitory model neural networks. Journal of Computational Neuroscience, 1(1):69-87, 1994.

[75] D. C. Somers, S. Nelson, and M. Sur. An Emergent Model of Orientation Selectivity in Cat Visual Cortical Simple Cells. J. Neurosci., 15:5448-5465, 1995.

[76] M L Steyn-Ross, D A Steyn-Rossand J W Sleigh, and D R Whiting. Theoretical predictions for spatial covariance of the electroencephalographic signal during the anesthetic-induced phase transition: Increased correlation length and emergence of spatial self-organization. Phys Rev E, 68:021902, 2003

[77] M. Stocker, M. Krause, and P. Pedarzani. An apamin-sensitive $\mathrm{ca}^{2+}$-activated $\mathrm{k}^{+}$current in hippocampal pyramidal neurons. Proc Natl Acad Sci U S A, 96:4662-4667, 1999.

[78] N V Swindale. The development of topography in the visual-cortex: A review of models. Network, 7:161-274, 1996.

[79] J. Tabak, W. Senn, M.J. O’Donovan, and J. Rinzel. Modeling of spontaneous activity in developing spinal cord using activity-dependent depression in an excitatory network. The Journal of Neuroscience, 20(8):3041, 2000.

[80] A.L. Taylor, G.W. Cottrell, and W.B. Kristan Jr. Analysis of oscillations in a reciprocally inhibitory network with synaptic depression. Neural computation, 14(3):561-581, 2002.

[81] M. Tsodyks, K. Pawelzik, and H. Markram. Neural networks with dynamic synapses. Neural Computation, 10(4):821-835, 1998.

[82] J.A. Varela, K. Sen, J. Gibson, J. Fost, LF Abbott, and S.B. Nelson. A quantitative description of short-term plasticity at excitatory synapses in layer $2 / 3$ of rat primary visual cortex. The Journal of neuroscience, 17(20):7926, 1997.

[83] H R Wilson and J D Cowan. Excitatory and inhibitory interactions in localized populations of model neurons. Biophys. J., 12:1-23, 1972.

[84] H R Wilson and J D Cowan. A mathematical theory of the functional dynamics of cortical and thalamic nervous tissue. Kybernetik, 13:55-80, 1973

[85] H.R. Wilson, R. Blake, and S.H. Lee. Dynamics of travelling waves in visual perception. Nature, 412(6850):907-910, 2001.

[86] K Zhang. Representation of spatial orientation by the intrinsic dynamics of the head-direction cell ensemble: a theory. J Neurosci, 16:2112-2126, 1996. 



\section{RECENT REPORTS}

12/22 Chebfun and Numerical Quadrature

Hale

Trefethen

12/23 Moment-based formulation of NavierMaxwell slip boundary con-

Reis

ditions for lattice Boltzmann simulations of rarefied flows in microchannels

12/24 Correspondence between one- and two-equation models for solute transport in two-region heterogeneous porous media

Davit

Wood

Debenest

Quintard

12/25 Rolie-Poly fluid flowing through constrictions: Two distinct instabilities

Reis

Wilson

12/26 Age related changes in speed and mechanism of adult skeletal

Collins-Hooper muscle stem cell migration

Woolley

Dyson

Patel

Potter

Baker

Gaffney

Maini

Dash

Patel

12/27 The interplay between tissue growth and scaffold degradation in

ODea engineered tissue constructs

Osborne

El Haj

Byrne

Waters

12/28 Non-linear effects on Turing patterns: time oscillations and

Aragon chaos.

Barrio

Woolley

Baker

Maini

12/29 Colorectal Cancer Through Simulation and Experiment

Kershaw

Byrne

Gavaghan

Osborne

12/30 A theoretical investigation of the effect of proliferation and adhe-

Mirams sion on monoclonal conversion in the colonic crypt

Fletcher

Maini

Byrne

12/31 Convergent evolution of spiny mollusk shells points to elastic en-

Chirat ergy minimum

Moulton

Shipman

Goriely

12/32 Three-dimensional oblique water-entry problems at small dead-

Moore rise angles

Howison

Ockendon

Oliver

12/33 Second weak order explicit stabilized methods for stiff stochastic

Abdulle 
12/37 The Dynamics of Liquid Drops and their Interaction with Solids of Varying Wettabilities

Sprittles

Shikhmurzaev

12/38 Dispersal and noise: Various modes of synchrony in ecological

Bressloff oscillators

Lai

12/39 Boundary conditions for free surface inlet and outlet problems

Taroni

Breward

Howell

Oliver

12/40 A Branch and Bound Algorithm for the Global Optimization of

Fowkes Hessian Lipschitz Continuous Functions

Gould

Farmer

12/41 The Orthogonal Gradients Method: a Radial Basis Functions

Piret Method for Solving Partial Differential Equations on Arbitrary Surfaces

12/42 Squeeze-Film Flow in the Presence of a Thin Porous Bed, with

Knox Application to the Human Knee Joint

Wilson

Duffy

McKee

12/43 Gravity-driven draining of a thin rivulet with constant width down

Paterson a slowly varying substrate

Wilson

Duffy

12/44 The 'Sticky Elastica': Delamination blisters beyond small deformations

Wagner

Vella

12/45 Stochastic models of intracellular transport

Bressloff

Newby

Copies of these, and any other OCCAM reports can be obtained from:

\section{Oxford Centre for Collaborative Applied Mathematics}

Mathematical Institute

24 - 29 St Giles'

Oxford

OX1 3LB

England

www.maths.ox.ac.uk/occam 